\title{
Characterization of Solidification Microstructures in Vacuum Arc Remelted Nickel Alloy 718
}

\author{
THOMAS A. IVANOFF, TREVOR J. WATT, and ERIC M. TALEFF
}

\begin{abstract}
Digital image analysis techniques were developed to autonomously characterize dendritic solidification features in microstructures of a nickel alloy 718 ingot produced by vacuum arc remelting (VAR). Automated macrophotography was implemented to image microstructures and produce large image montages of etched ingot cross sections. Two image analysis techniques, particle identification and two-point correlation, were applied to autonomously measure primary dendrite arm orientation and secondary dendrite arm spacing from the image montages. Particle identification measured individual dendritic features. The two-point correlation technique measured averaged feature values over defined image areas. These methods are described and compared. Melt pool profile and solidification time histories were reconstructed from primary dendrite arm orientation and secondary dendrite arm spacing measurements, respectively. These characterization techniques provide two new methods of reconstructing melt pool profiles and solidification times in cast ingots. The information produced is expected to be useful in the validation of computational models for solidification during VAR, similar remelting processes, and general casting processes that produce dendritic solidification features.
\end{abstract}

https://doi.org/10.1007/s11663-019-01530-2

(C) The Author(s) 2019

\section{INTRODUCTION}

CAST-AND-Wrought nickel alloy 718 is the most used superalloy in the aerospace industry ${ }^{[1-3]}$ because of its useful combination of mechanical properties $^{[4-11]}$ and manufacturability. ${ }^{[9,11,12]}$ If processed appropriately, this material can exhibit good creep strength, oxidation resistance, and microstructural stability up to $650{ }^{\circ} \mathrm{C} .^{[4,5,8,10]}$ These mechanical properties are derived from a uniform distribution of coherent $\gamma^{\prime \prime}$ $\left(\mathrm{Ni}_{3} \mathrm{Nb}\right)$ intermetallic precipitates distributed throughout the $\gamma$-nickel lattice. ${ }^{[4,5,13,14]}$ Formation of the $\gamma^{\prime \prime}$ phase is promoted by niobium in alloy 718 ; the nominal composition of alloy 718 is provided in Table $\mathrm{I}^{[5]}$ Mechanical properties depend heavily on the microstructure produced by liquid metal processing (LMP), i.e., casting. Defects formed during LMP often cannot be remediated by subsequent processing steps such as homogenization and mechanical deformation. ${ }^{[15-21]}$ Some defects can cause non-uniform

THOMAS A. IVANOFF is with Materials Mechanics \& Tribology, Sandia National Laboratories, P.O. Box 5800, Albuquerque, NM, 87185-0889. Contact e-mail: tivanof@sandia.gov TREVOR J. WATT is with Avid R\&D LLC, Jollyville Rd., 202376, Austin, TX, 78712. ERIC M. TALEFF is with the Department of Mechanical Engineering, The University of Texas at Austin, Austin, TX, 78712.

Manuscript submitted September 13, 2018.

Article published online February 12, 2019. precipitation of $\gamma^{\prime \prime}$ during artificial aging, which creates regions with poor mechanical strength. ${ }^{[22-24]}$ For critical applications in gas-turbine engines, these weak regions may produce catastrophic failures, which cannot be tolerated. Consequently, inferior ingots must be melted again, which is costly and time consuming.

Typical LMP of alloy 718 often includes vacuum-induction melting (VIM) followed by electro-slag remelting (ESR) and/or vacuum arc remelting (VAR). ${ }^{[25-29]}$ VAR, the process shown schematically in Figure 1, is often the last step in LMP. VAR improves ingot quality by removing undesirable oxides and volatile gases and by refining solidification microstructures. ${ }^{[25]}$ VAR uses a direct current to melt an electrode, typically an ingot produced by VIM or ESR, which then solidifies in a water-cooled crucible. Solidification begins on the chilled stool at the bottom of the crucible. As molten metal from the electrode falls into the crucible, a molten pool of metal, called the melt pool, is formed. The size and geometry of the ingot, the electrode current, and the melt rate all influence the melt pool profile and the local solidification rate of the material. The melt pool profile is the average shape of the interface between the melt pool (liquidus isotherm) and the solidified material (solidus isotherm). Local solidification time is typically defined by the time required to reach the solidus temperature from the liquidus temperature. ${ }^{[30]}$ The melt pool profile and local solidification time are important to control because they influence the likelihood of 
Table I. The Specified Elemental Composition Limits of Nickel Alloy 718 Are Provided in Weight Percent ${ }^{[5]}$

\begin{tabular}{llccccccrrr}
\hline Element & $\mathrm{Ni}$ & & $\mathrm{Cr}$ & & $\mathrm{Fe}$ & & $\mathrm{Nb}$ & $\mathrm{Mo}$ & $\mathrm{Ti}$ & $\mathrm{Al}$ \\
\hline Wt pct & $50-55$ & & $17-21$ & & bal. & & $4.75-5.5$ & $2.8-3.3$ & $0.6-1.15$ & $0.2-0.8$ \\
\hline Element & $\mathrm{Co}$ & $\mathrm{C}$ & & $\mathrm{Mn}$ & $\mathrm{Si}$ & $\mathrm{P}$ & $\mathrm{S}$ & $\mathrm{B}$ & $\mathrm{Cu}$ \\
\hline Wt pct & $<1$ & $<0.08$ & $<0.35$ & $<0.35$ & $<0.015$ & $<0.015$ & $<0.006$ & $<0.3$ \\
\hline
\end{tabular}

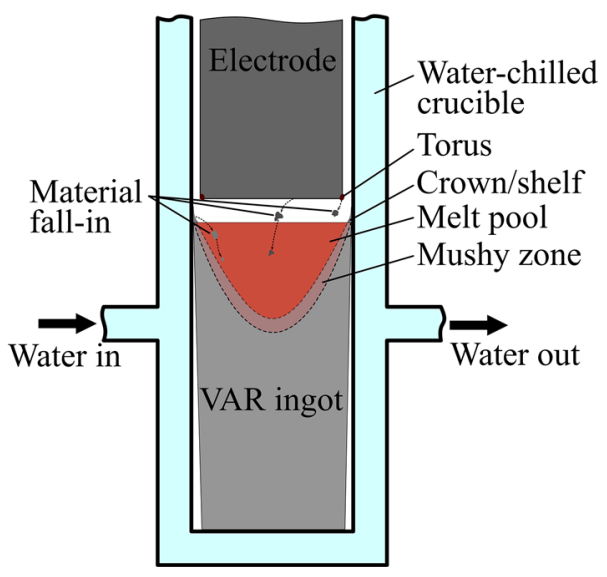

Fig. 1-This schematic depicts the vacuum arc remelting (VAR) process and identifies regions of interest.

defects forming during solidification. Defects commonly form during VAR by macro-segregation along the melt pool profile, i.e., freckle channels and solidification white spots, and by unmelted material entering the melt pool, i.e., discrete white spots and dendritic white spots. $^{[11,14,22,25,27-29,31-43]}$ When the melt pool is too shallow or too deep, the formation of these defects is more likely. ${ }^{[1,14,29,33]}$ After forming, these segregation defects cannot be easily remediated by subsequent homogenization heat treatments or hot forging and are detrimental to the final wrought product. ${ }^{[15-24]}$

Computational models have been produced to simulate solidification during VAR and ESR. Manufacturers use these models to determine processing conditions for control of the microstructures produced in solidified ingots. $^{[25,26,33,44-50]}$ To this end, manufacturers are interested in predicting and controlling the melt pool profile and solidification time because these both influence the microstructure of the solidified ingot. ${ }^{[4,51]}$ Measurement of melt pool profiles and local solidification times are needed to validate the computational models for remelting. Currently, in situ measurements of melt pool profiles and local solidification times during remelting are not feasible. These measurements must be acquired from solidified ingots after remelting is completed. The previous standard for these measurements required the use of melt pool markers, features intentionally introduced during the remelting process to decorate the melt pool profile at distinct locations within an ingot. ${ }^{[44,46,52-55]}$ Melt pool profiles are measured from these markers in sections extracted from a solidified ingot. Melt pool profiles cannot be measured continuously throughout a solidified ingot using pool marking techniques because the markings can only be added at a few select locations during the remelting process.

This study proposes the use of microstructural features intrinsic to dendritic solidification to determine melt pool profiles and local solidification times as an alternative to marking techniques. Melt pool profiles can be estimated from primary dendrite arm orientations (PDAO) in regions of directional solidification because primary dendrite arms are oriented orthogonally to the melt pool profile, to a reasonable approximation. ${ }^{[30]}$ Local solidification times can be calculated indirectly from measurements of secondary dendrite arm spacing (SDAS) in solidified ingots after remelting is completed. Measurements may be acquired from ingot cross sections etched to reveal these dendritic features. Traditionally, SDAS is manually measured from such images, which is time consuming and can often be used only to provide measurements at a few locations within an ingot. Equation 1 presents a relationship used to calculate local solidification time from SDAS. ${ }^{\text {30] }}$

$$
\lambda=5.5 M\left(t_{f}\right)^{1 / 3}
$$

Here, $\lambda$ is $\operatorname{SDAS}, M$ is a material-dependent parameter, and $t_{f}$ is local solidification time. According to this relationship, the cube root of local solidification time is proportional to SDAS and can be calculated if the appropriate material-dependent parameter, $M$, is known. For alloy 718, the material-dependent parameter, $M$, was calculated to be $2.74 \mu \mathrm{m} / \mathrm{s}^{1 / 3}$ based on the data presented by Rappaz and Boettinger ${ }^{[56]}$ and Boettinger et al. ${ }^{[57]}$

For this study, solidification microstructures were imaged using automated macrophotography and characterized throughout slabs extracted from an alloy 718 ingot produced by VAR. PDAO and SDAS were measured from montage images of microstructure using two automated, digital image analysis techniques: (1) particle identification and (2) a two-point correlation function. The particle-identification algorithms used were first introduced by Watt et al. ${ }^{[5]}$ and detect individual primary dendrite arms in the microstructure. The two-point correlation function method generated statistical analyses of microstructure. Fourier analysis was then used to measure PDAO and aide in the measurement of SDAS. Melt pool profiles and local solidification times were estimated using data from both techniques. SDAS measurements from the automated 
techniques were also compared to manual measurements. The techniques developed are demonstrated on an alloy 718 material produced by VAR, but these techniques are generally applicable to other LMP technologies that produce dendritic microstructures.

\section{MATERIALS}

Material characterized in this study was extracted from a laboratory-scale alloy 718 ingot that initially measured $212 \mathrm{~mm}$ in diameter and $406 \mathrm{~mm}$ in height. This ingot was produced at Los Alamos National Laboratory by VAR. A single slab, approximately $28 \mathrm{~mm}$ thick, was sectioned $2.54 \mathrm{~mm}$ off center from this ingot along the casting direction. This slab was further sectioned in half, perpendicular to the casting direction, to facilitate handling. The schematic in Figure 2(a) illustrates the extraction process. After extraction, each slab was precision ground to a 35 RA ( $\mu$ in) finish and a flatness tolerance of \pm 0.01 in $(0.254 \mathrm{~mm})$ to reveal the ingot centerline and then chemically etched to reveal microstructure. A segregation etch, Canada's etchant ( 8 parts $\mathrm{H}_{2} \mathrm{O}, 2$ parts $\mathrm{H}_{2} \mathrm{SO}_{4}, 2$ parts $\mathrm{HF}$, and 1 part $\left.\mathrm{HNO}_{3}\right)$, applied at or above $70{ }^{\circ} \mathrm{C},{ }^{[59]}$ revealed the dendritic microstructure. Figures 2(b) and (c) present the microstructure of the two prepared slabs. The $\mathrm{V}$-shaped shrinkage pipe and shrinkage cavity located at the top of the ingot, as shown in Figure 2(b), were created at the end of the melt when the electrode current was stopped. The ingot was sectioned and precision ground by ATI Precision Finishing (Rochester, PA) and chemically etched by Special Metals Corp. Each slab was cleaned with soapy water and a stiff nylon brush prior to characterization.

\section{EXPERIMENTAL METHODS}

\section{A. Imaging Instrumentation}

A Canon ${ }^{\mathrm{TM}}$ EOS 60D digital single-lens reflex camera equipped with a Sigma ${ }^{\mathrm{TM}} 105 \mathrm{~mm}$ F2.8 EX DG OS HSM macro lens acquired images of microstructure. Imaging was conducted at approximately 1:1 image

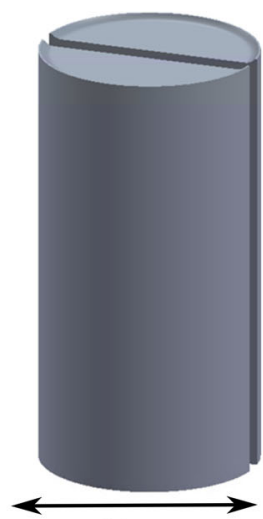

$21 \mathrm{~cm}$

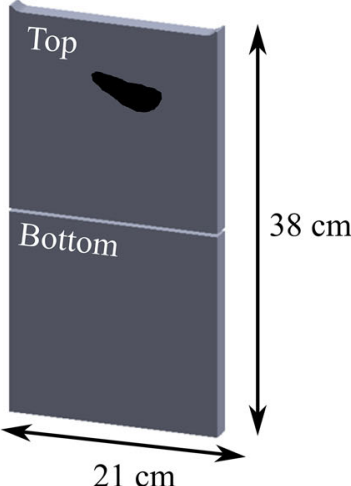

(a)

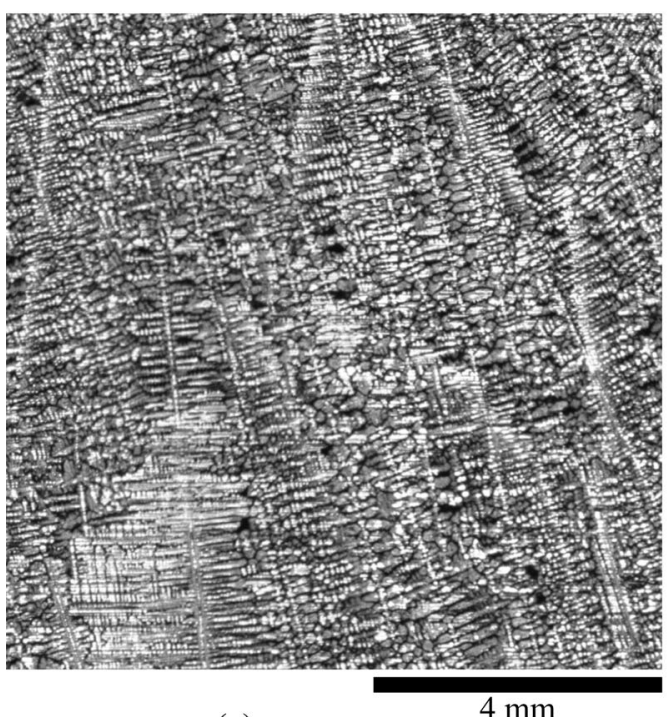

(c)
$4 \mathrm{~mm}$

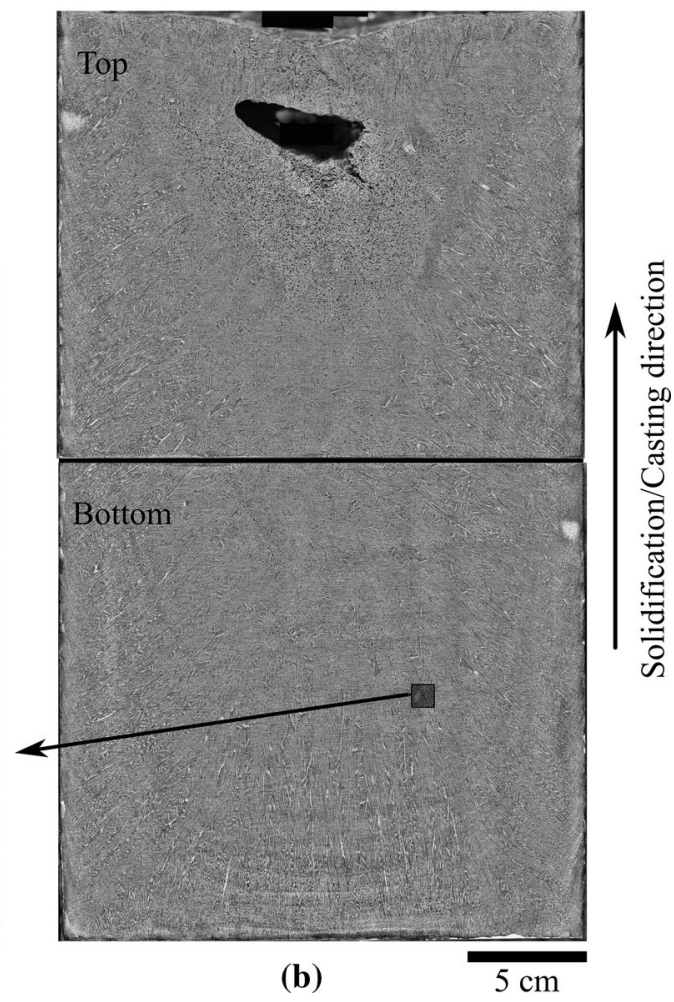

Fig. 2-(a) The extraction of the top and bottom slabs from the VAR alloy 718 ingot produced by Los Alamos National Laboratory is shown. (b) Montage images of each slab are shown. (c) An expanded region of the montage images that demonstrates the image resolution is shown. Slab surfaces were prepared for imaging by sectioning, grinding, etching (Canada's etchant ${ }^{[59]}$ ), and cleaning. 
magnification, i.e., the image size projected onto the camera sensor is the same as the size of the object viewed. Lens distortion was experimentally determined to be on the order of the pixel size and was, thus, negligible. This system provided a maximum resolution of approximately $4.3 \mu \mathrm{m} /$ pixel. This resolution is sufficient to identify individual dendrites, as demonstrated by Figure 2(c). Two strobe units provided uniform illumination across each slab during imaging. A circular polarizing filter attached to the lens reduced glare for improved image quality. Experiments determined macrophotography settings to provide the best balance of image clarity and depth of focus. These were an F-stop of 8 , an exposure time of $1 / 250$ seconds, and an ISO number of 100 .

A robotic stage was constructed to autonomously image each slab. Two stepper motors controlled motion of the macrophotography equipment to acquire images in sequential steps per the schematic presented in Figure 3. Approximately 20 pct linear overlap was provided between adjacent images to enable subsequent digital stitching of images into a single montage image of each slab. The complete areas of the top and bottom slabs were imaged separately with 250 and 204 individual macrophotographs, respectively. Phase detection auto focus was used to account for surface roughness across the slab. Magnification varied from approximately 4.3 to $4.4 \mu \mathrm{m} /$ pixel over the top slab and from 4.4 to $4.5 \mu \mathrm{m} /$ pixel over the bottom slab. The slabs were supported and leveled within the robotic stage prior to imaging using three adjustable mounts. ${ }^{[60]}$

\section{B. Digital Image Processing}

Image data were initially acquired in the raw CR2 native Canon ${ }^{\mathrm{TM}}$ image format and then processed into the uncompressed 8-bit grayscale TIFF (Tagged Image File Format) image format. Montage images were

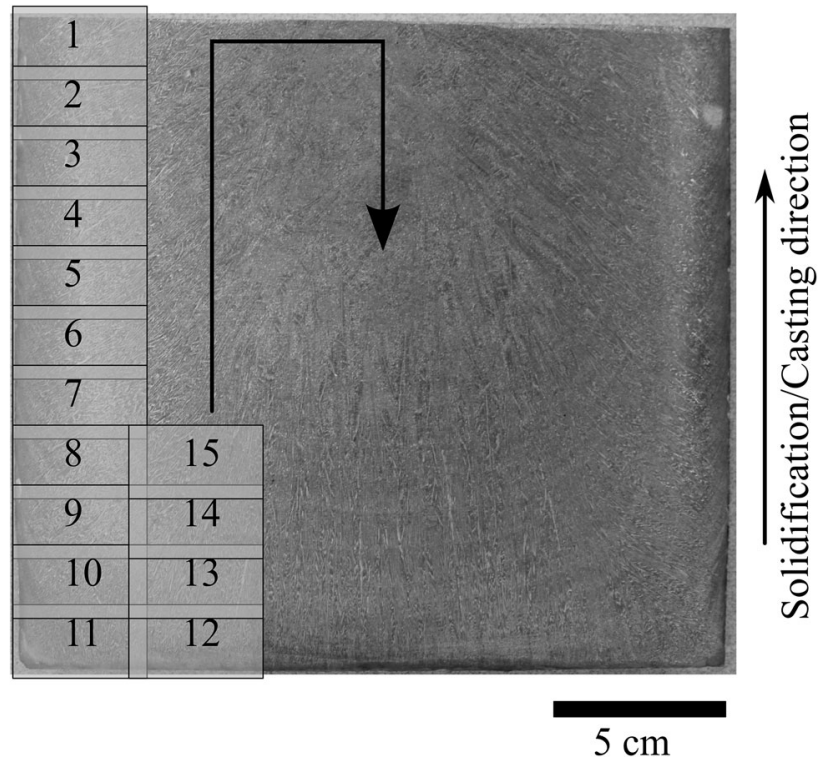

Fig. 3-The image acquisition and stitching patterns are demonstrated across a slab surface. created from these TIFF images using the grid/collection stitching plugin contained within the Fiji distribution of ImageJ. ${ }^{[61]}$ The TIFF images were manipulated as follows prior to stitching. First, a CLAHE (contrast limited adaptive histogram equalization) algorithm ${ }^{[62]}$ was applied to stretch local contrast across the 8-bit file depth and improve feature recognition during stitching. Second, images were reduced in size by approximately 50 pct using bilinear scaling to prevent the montage image from exceeding the $1 \mathrm{~GB}$ file-size limitation of the Fiji software. The image resolution was reduced to approximately $9 \mu \mathrm{m} /$ pixel, which is sufficient for feature analysis.

Computerized image analysis software is not sophisticated enough to identify primary and secondary dendrite arms directly from the montage images. Therefore, the montage images were processed into a binary image format that the computerized image analysis techniques are capable of handling. Montage images were binarized in Matlab ${ }^{\mathrm{TM}[63]}$ using a threshold value of 127 on an 8-bit scale. Pixels with an intensity value below this threshold were converted to a value of 0 (black) and those above were converted to a value of 1 (white). With respect to the material microstructures, inter-dendritic regions were processed into black pixels and dendrite arms were generally processed into white pixels.

\section{Particle-Identification Technique}

The particle-identification algorithms and procedures used in this study are an extension of those presented by Watt et al. ${ }^{[58]}$ This technique relies on the accurate identification of primary dendrite arms as individual particles to measure primary dendrite arm orientation (PDAO) and secondary dendrite arm spacing (SDAS). The particle-identification algorithms contained within Fiji software were used to identify particles from binary montage images. This algorithm locates and identifies discrete areas of continuous white pixels and labels those areas as individual particles; it ignores regions of continuous black pixels. However, software identification of features, i.e., particles, from the as-converted binary image was poor because microstructural features often appear connected. To address this issue, each binary image was further processed using two morphological erosion operations to eliminate interconnections and thus improve feature identification.

After particle identification was completed, particles not likely to represent primary dendrite arms were filtered from the data set. Particles were filtered based on length, aspect ratio, and variation of pixel values across the particle. The parameters determined to produce useful filtering and applied in this study are listed in Table II. After applying the filters described in Table II, a subset of remaining particles did not correspond with primary dendrite arms. These were attributed to the unintended detection of secondary dendrite arms as primary arms. The secondary arms are approximately orthogonal to primary arms. These misidentifications were corrected by rotating the particle orientation 90 deg to correspond with the primary dendrite from 
Table II. The Filter Parameters Used to Remove Particles Not Likely to Represent Primary Dendrite Arms for the Particle-Identification Technique are Provided

\begin{tabular}{|c|c|c|}
\hline Filter & Function & Removal Criterion \\
\hline Length & $\begin{array}{l}\text { Removed particles deemed too short or too long to represent a } \\
\text { primary dendrite arm }\end{array}$ & $\begin{array}{l}\text { Long axis shorter than } 22 \text { pixels } \\
\text { Long axis longer than } 5000 \text { pixels }\end{array}$ \\
\hline Aspect ratio & Removed particles with low aspect ratios & Aspect ratio less than 5 \\
\hline $\begin{array}{l}\text { Variation of } \\
\text { pixel } \\
\text { intensity } \\
\text { along par- } \\
\text { ticle long } \\
\text { axis }\end{array}$ & $\begin{array}{l}\text { Removed particles with a large relative standard deviation } \\
\text { Relative standard deviation was calculated by: (1) measuring pixel } \\
\text { intensities along the particle long axis and (2) dividing the stan- } \\
\text { dard deviation of the intensities by the mean of the intensities }\end{array}$ & A relative standard deviation greater than 0.5 \\
\hline
\end{tabular}

which the identified secondary dendrite grew. The few remaining outlier particles were filtered by eliminating those with a long-axis orientation at least two standard deviations from the average orientation angle measured in a circular region with a radius of 500 pixels. This final filtering reduced measurement noise in regions with a consistent PDAO without significantly affecting regions with a random distribution of orientations.

Figure 4 presents primary dendrite arms identified from an image as (a) lines overlaid on the original image and (b) as lines only. Figure 4(a) demonstrates that this particle-identification technique identified many, but not all, of the primary dendrite arms visually observed in this microstructure. However, a sufficient number of primary dendrite arms were identified to quantitatively characterize PDAO.

Figure 5 illustrates the measurement of SDAS using the previously identified primary dendrite arms. SDAS was measured from the change in image grayscale intensity along lines running parallel and directly adjacent to each identified primary dendrite arm; see Figure 5(a). The distance between peaks in the image intensity profile was interpreted as the SDAS; see Figure 5(b). SDAS was measured for analysis lines offset by 8 to 16 pixels from each primary dendrite arm, which corresponds to a range of 72 to $144 \mu \mathrm{m}$. The offset line with the smallest standard deviation in SDAS was retained. If less than three peaks were identified along a primary dendrite arm, no value for SDAS was recorded. These operations were automated in a Matlab ${ }^{\text {TM }}$ code.

Two filters were applied to the automated SDAS measurements. The first filter removed measurements that were at least two standard deviations away from the average in a circular region with a radius of 500 pixels. The second filter removed measurements from regions that produced unrealistically large values of SDAS; values greater than $270 \mu \mathrm{m}$. This upper bound on SDAS was determined by manually measuring SDAS in several regions with clearly visible secondary dendrite arms, which produced an average measurement of approximately $100 \mu \mathrm{m}$.

\section{Two-Point Correlation Technique}

The two-point correlation function implementation used in this study is based on that developed by Chang. ${ }^{[64]}$ The resulting code was used to calculate statistical information from image data to measure PDAO and

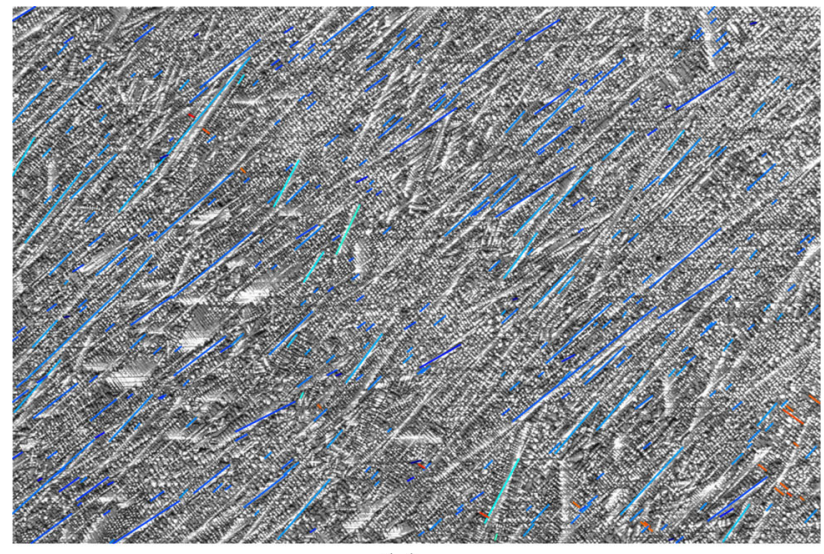

(a)

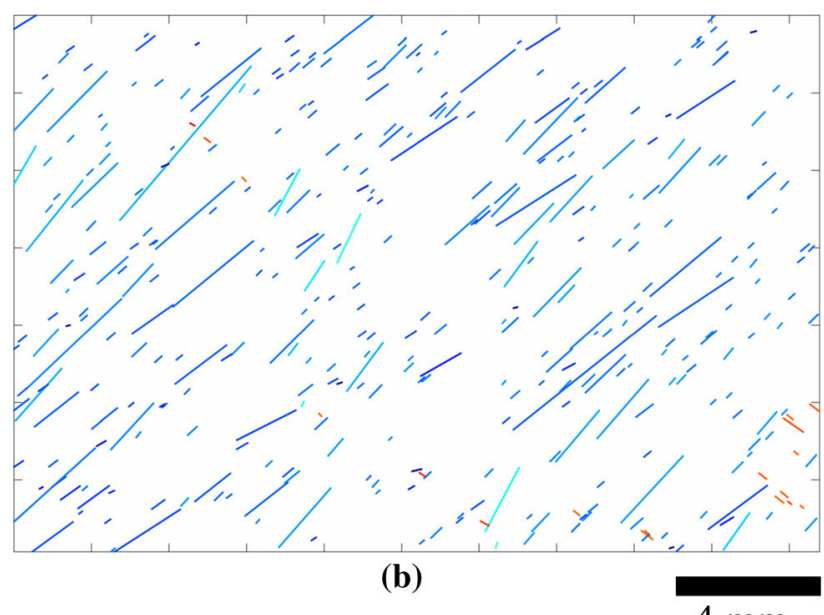

$4 \mathrm{~mm}$

Fig. 4-Primary dendrite arms identified from microstructure using the particle-identification technique are shown $(a)$ overlaid on the image data and $(b)$ alone. These particles, i.e., primary dendrite arms, are presented after filtering.

SDAS. The two-point correlation function, $P_{i j}(r, \theta)$, is defined as the probability that a line of length $r$ at angle $\theta$ will have start and end points in "phases" $i$ and $j$, respectively. The orientation-averaged probability function, $P_{i j}(r)$, is calculated by integrating $P_{i j}(r, \theta)$ over the orientation angle, $\theta$, from 0 to $\pi / 2 .^{[64-66]} P_{i j}(r)$ is defined as the average probability that a line with length $r$ and endpoints in "phases" $i$ and $j$ exists. For the purpose of 
this study, only two "phases" are defined: dendrite arms (white in the binary images) are defined as phase 1, and inter-dendritic regions (black in the binary images) are defined as phase 2. The two-point correlation function can provide four different probabilities: $P_{11}(r), P_{12}(r)$, $P_{21}(r)$, and $P_{22}(r)$. Because only dendrite-to-dendrite statistics are relevant to calculating PDAO and SDAS, only $P_{11}(r)$ was calculated for this study.

Two-point correlation function analysis was implemented in Mathematica ${ }^{\mathrm{TM}[67]}$ on the as-converted binary montage images; no morphological processing was necessary prior to analysis. This implementation used a

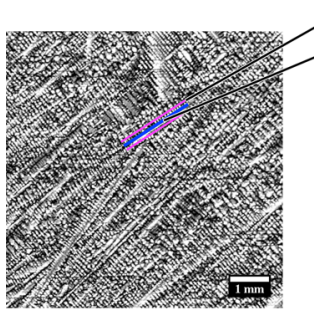

(a)
SDAS analysis line 1

Primary dendrite arm analyzed

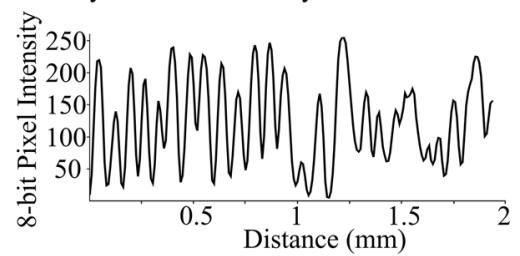

(b)

Fig. 5-The measurement of SDAS is illustrated using the particle-identification technique. (a) An individual primary dendrite arm is identified and presented with its corresponding SDAS analysis lines. (b) The image intensity profile along one of the analysis lines is shown.

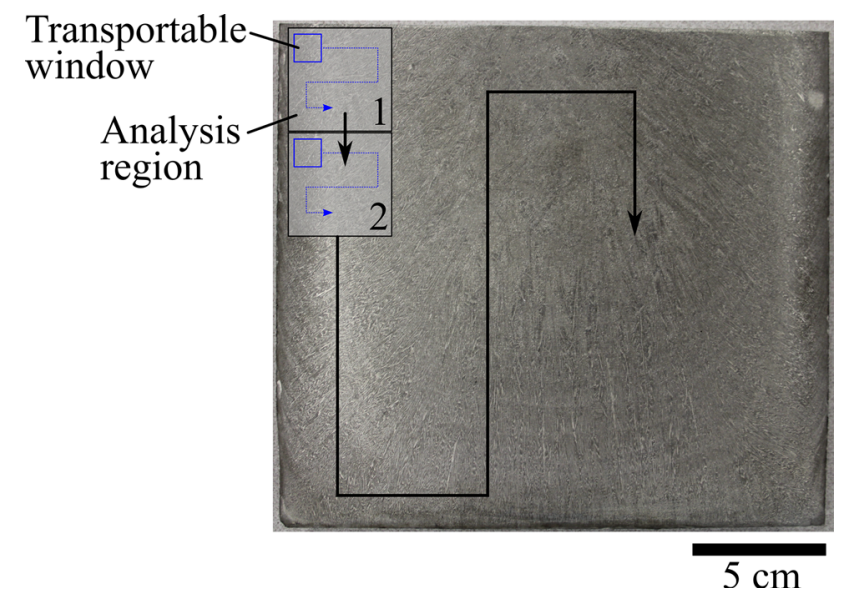

Fig. 6-The sequential motion of the analysis region used for the two-point correlation technique is shown across a montage image. Two-point correlation functions, $P_{11}(r)$, are calculated in each analysis region using the transportable window to quantify the average orientation of dendritic features.

Table III. The Rules for Calculating the $P_{11}(r, \theta)$ Array Inside the Transportable Window Using the Two-Point Correlation Technique

\begin{tabular}{lccc}
\hline $\begin{array}{l}\text { Correlation } \\
\text { Type }\end{array}$ & $\begin{array}{c}\text { Central Pixel } \\
\text { Value }\end{array}$ & $\begin{array}{c}\text { Other Pixel } \\
\text { Values }\end{array}$ & $\begin{array}{c}\text { Integer Value } \\
\text { Assigned }\end{array}$ \\
\hline 1 to 1 & 1 & 1 & 1 \\
0 to 1 & 0 & 1 & 0 \\
& 1 & 0 & 0 \\
\hline
\end{tabular}

transportable window to compute the two-point correlation function from a selected analysis region. Note that the sizes of both the analysis region and the transportable window must be appropriately scaled to the microstructural feature size of interest and the image resolution. For this study, the best balance of data averaging, retention of fine feature detail, and computational processing time was achieved using a $512 \times 512$ pixel analysis region and a $256 \times 256$ pixel transportable window. Each montage image was analyzed by sequentially moving the analysis region across the entire image; Figure 6 illustrates this process. Positions within the transportable window were assigned integer values of 1 or 0 according to Table III. The individual $P_{11}(r, \theta)$ arrays from each analysis region were summed to calculate $P_{11}(r)$, which describes the two-point correlation function for the entire analysis region. Each value in $P_{11}(r)$ was divided by the maximum value in the array to produce the percent probability of a dendrite-to-dendrite interaction. These probability arrays were visualized as 8-bit grayscale images; bright and dark regions represent high and low probabilities, respectively.

Figures 7(a) and (b) present one analysis region from a microstructural image and the two-point correlation data it produced, respectively. The two-point correlation data were analyzed using a two-dimensional Fast Fourier Transform (FFT) implemented in Matlab ${ }^{\mathrm{TM}}$ to determine PDAO and secondary dendrite arm orientation (SDAO). Two-point correlation data were windowed using a Hann function ${ }^{[68]}$ to prevent image artifacts during application of the FFT. Figure 7(c) presents the power spectrum calculated from the FFT of Figure 7(b) on a base-10 logarithmic scale to improve image contrast; image data are rotated $90 \mathrm{deg}$ with respect to the data shown in Figures 7(a) and (b). The PDAO, SDAO, and orientation of scratches on the surface of the specimen are revealed as the streaks labeled in Figure 7(c).

PDAO and SDAO were measured from the FFT data using an angular filter. The angular filter is equal in size to the FFT data and constructed from a zero-valued background and two symmetric non-zero Gaussian distributions positioned near its center. The filter values within the Gaussian distribution have a peak value of 1 and decline toward zero as described by the following equations:

$$
\begin{gathered}
G(x, y)=\left(1 / 2 \pi \sigma_{x} \sigma_{y}\right) e^{(-f(x, y))}, \\
f(x, y)=\frac{\left(x-\mu_{x}\right)^{2}}{2 \sigma_{x}^{2}}+\frac{\left(y-\mu_{y}\right)^{2}}{2 \sigma_{y}^{2}} .
\end{gathered}
$$

Here $G(x, y)$ is the Gaussian distribution; $\sigma_{x}$ is the standard deviation along the x-axis; $\sigma_{y}$ is the standard deviation along the $\mathrm{y}$-axis; $\mu_{x}$ is the mean along the $\mathrm{x}$-axis; and $\mu_{y}$ is the mean along the y-axis. For this study, $\mu_{x}$ and $\mu_{y}$ were set to $0, \sigma_{x}$ was set to 2.5 , and $\sigma_{y}$ was set to 0.25 . Figure $7(\mathrm{~d})$ presents the angular filter, oriented at $0 \mathrm{deg}$, at its full size; the Gaussian distributions are shown in white. The center of the angular filter is enlarged in 


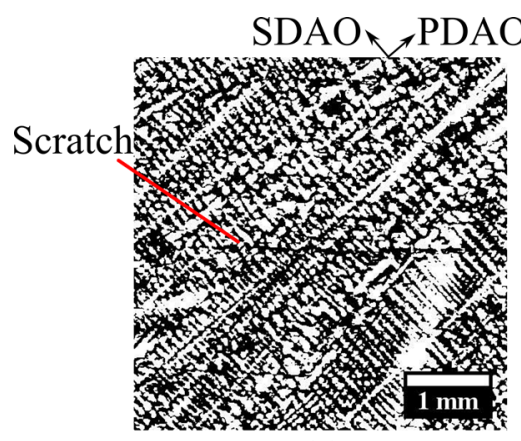

(a)

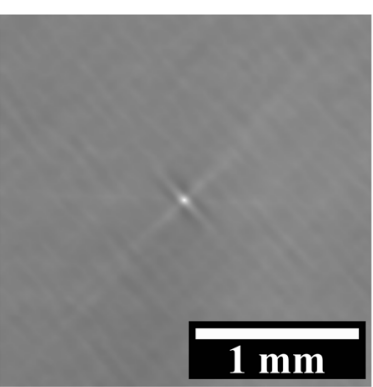

(b)

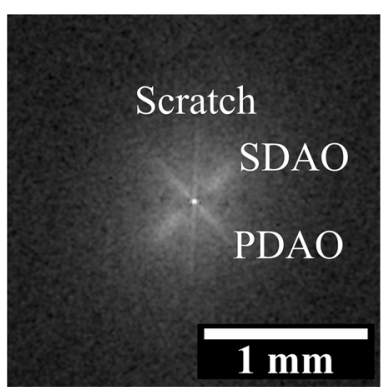

(c)

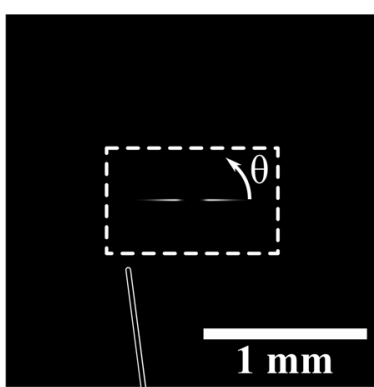

(d)

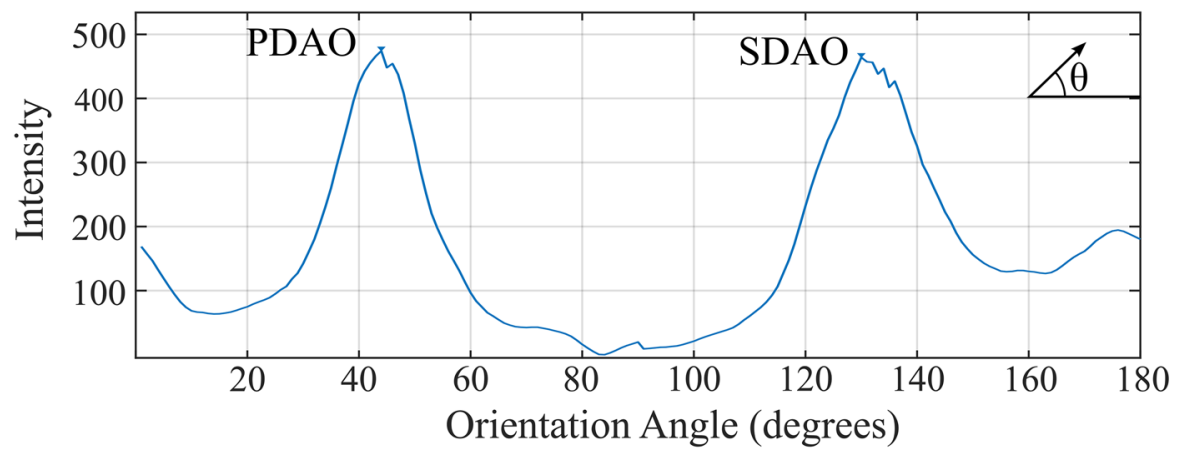

(e)

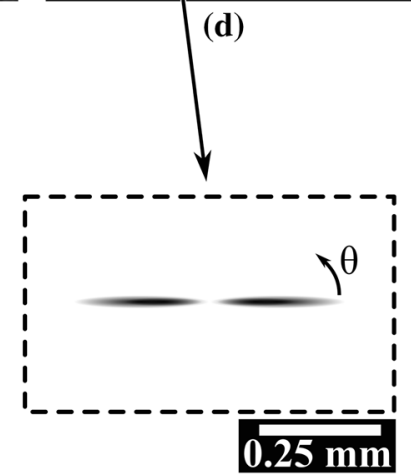

(f)

Fig. 7-Measurement of PDAO using the two-point correlation technique is illustrated. (a) Microstructure is processed into a binary image format; note the SDAO and PDAO. (b) The two-point correlation data are presented as an 8-bit grayscale image. (c) The power spectrum calculated from the two-dimensional FFT of the two-point correlation data is shown. Note that the power spectrum is presented on a base-10 logarithmic scale and is rotated $90 \mathrm{deg}$ with respect to the microstructure and the two-point correlation data. Shown in $(d)$ and $(f)$ is the angular filter, oriented at $0 \mathrm{deg}$, used to calculate the intensity of each orientation angle. Note that the angular filter is shown enlarged and with inverted colors in (f) for visual clarity. (e) The intensity of each orientation angle is plotted; note the peaks associated with the PDAO and the SDAO.

Figure 7(f) to highlight the shape of the Gaussian distributions; color is inverted from (d) for easier visualization. The angular filter was applied to orientations from 0 to 179 deg at 1 deg increments. The angular data can be viewed by plotting the summed intensity values against orientation angle, as shown in Figure 7(e). Peaks in the data represent the PDAO and SDAO angles. Additional information on the PDAO and SDAO peak measurement process is provided in Reference 60.

SDAS was measured from the periodicity of image intensity in the two-point correlation data along the PDAO. Pixel intensity in the two-point correlation data was first summed along columns perpendicular to the PDAO. Figure 8 presents a typical result from a $128 \times 128$ pixel region of data. Figure 8(c) shows summed intensity as a function of distance along the PDAO. The average spacing between peaks was calculated and taken as the SDAS. A minimum of 4 peaks was stipulated to define a valid measurement.

\section{EXPERIMENTAL RESULTS}

\section{A. Primary Dendrite Arms and Melt Pool Profiles}

Primary dendrite arm orientation (PDAO) and melt pool profile estimates acquired from the ingot slabs using the particle-identification and two-point correlation techniques are presented in Figure 9. Figures 9(a) and (b) present results from the particle-identification technique. Figures 9(c) and (d) present results from the two-point correlation technique.

Figure 9(a) contains 55,640 individual primary dendrite arms; the color of each primary dendrite arm describes its orientation within the ingot per the legend provided. The number of primary dendrite arms identified by particle identification is high along the left, right, and bottom of the ingot. In these regions, directional solidification occurred, and the primary dendrite arms share similar orientations. On the left surface of the ingot, the PDAO is approximately $25 \mathrm{deg}$, as referenced to the legend in Figure 9(a). This angle increases to approximately $50 \mathrm{deg}$ at $40 \mathrm{~mm}$ from the left surface of the ingot. On the right side of the ingot, the average PDAO decreases from approximately $156 \mathrm{deg}$ at the right surface to approximately $125 \mathrm{deg}$ at $40 \mathrm{~mm}$ from the right surface of the ingot. Along the bottom of the ingot, primary dendrite arms take an angle of approximately $90 \mathrm{deg}$. In the center of the ingot, where equiaxed solidification occurs, primary dendrite arms appear randomly oriented. Primary dendrite arms are identified less frequently at the center compared to the sides of the ingot. The geometry of the primary dendrite arms evident in the equiaxed region likely interferes with the particle-identification methodology. Primary dendrite arms in the equiaxed region often pass through the sectioning plane, instead of lying in that plane, preventing them from being readily 
identified. Along the sides of the ingot, a large fraction of primary dendrite arms lie approximately within the sectioning plane, making them readily identifiable.

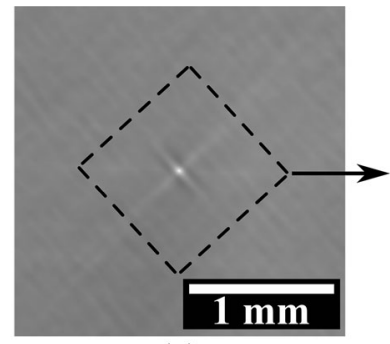

(a)

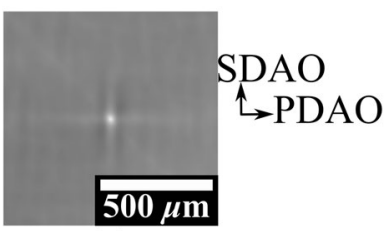

(b)

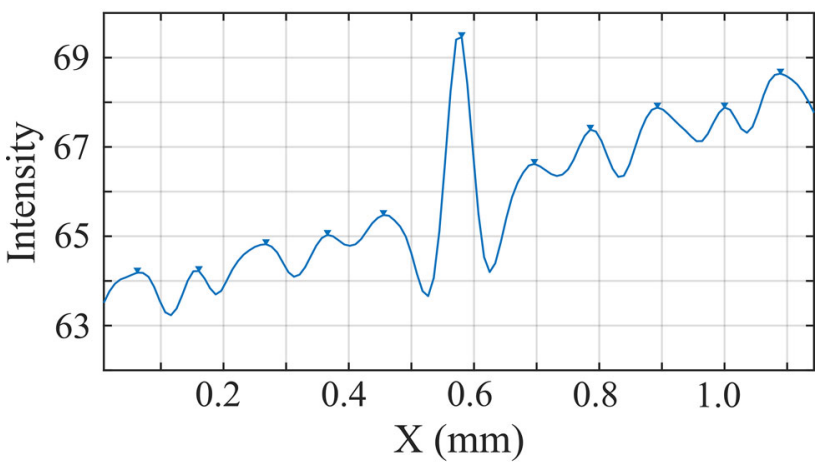

(c)

Fig. 8-Measurement of SDAS is demonstrated using the two-point correlation technique. (a) The two-point correlation data are shown. (b) A $256 \times 256$ pixel square region is selected and rotated so that the PDAO is oriented at $0 \mathrm{deg}$. (c) Image intensity is summed along the SDAO and plotted along the PDAO (X direction); the distance between peaks describes the SDAS.
The number of primary dendrite arms identified using particle identification were compared to the number identified manually in six $20 \mathrm{~mm}$ square regions selected from the lower left and right corners of the top slab and from all four corners of the bottom slab. Each region contained a minimum of 50 primary dendrite arms detected using particle identification. The percentage of manually identified primary dendrite arms also correctly identified by the particle-identification technique is 52 pct on average, with minimum and maximum percentages of 32 pct and 67 pct among the six regions analyzed. The particle-identification technique misidentified features as primary dendrite arms rarely, less than 1 in 30, not counting secondary dendrite arms identified and corrected to provide PDAO, as previously described.

Figure 9(b) presents the melt pool profiles estimated from primary dendrite arm orientations in Figure 9(a). Melt pool profiles were estimated using a streamline function in Matlab ${ }^{\mathrm{TM}}$. These profiles were started on the left and right surfaces of the ingot at several locations along the ingot height and were calculated toward the center of the ingot. As shown in Figure 9(b), the profiles were stopped $60 \mathrm{~mm}$ into the ingot from either surface to avoid the equiaxed region at the center of the ingot. Melt pool profiles cannot be readily determined from the inconsistently oriented primary dendrite arms in the equiaxed region. Note that the melt pool profiles are not smooth and often exhibit sudden changes in slope. These irregularities along each predicted melt pool may be a result of the estimation technique. Because the streamline function relies on individual PDAO measurements, a single erroneous data point can produce large changes to the slope. This is especially likely

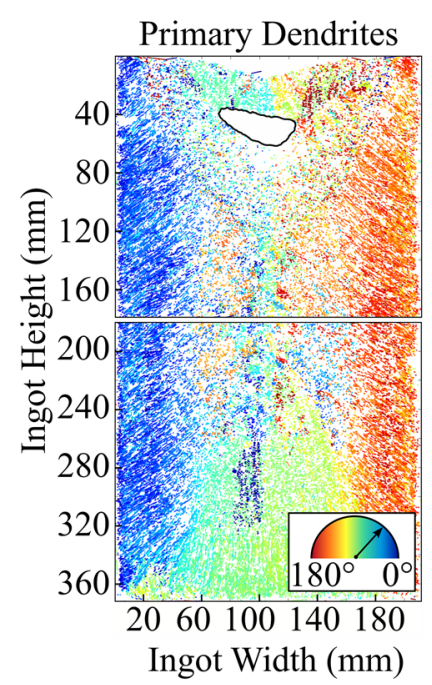

(a)

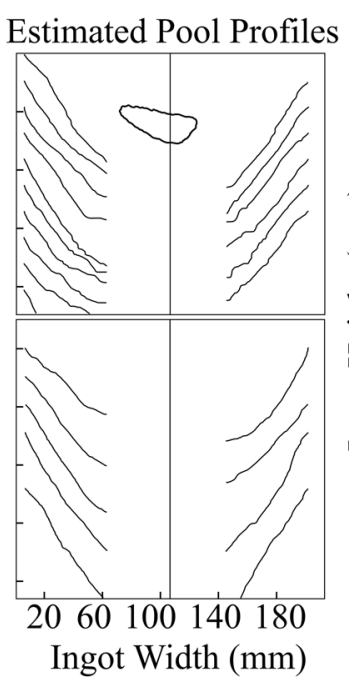

(b)

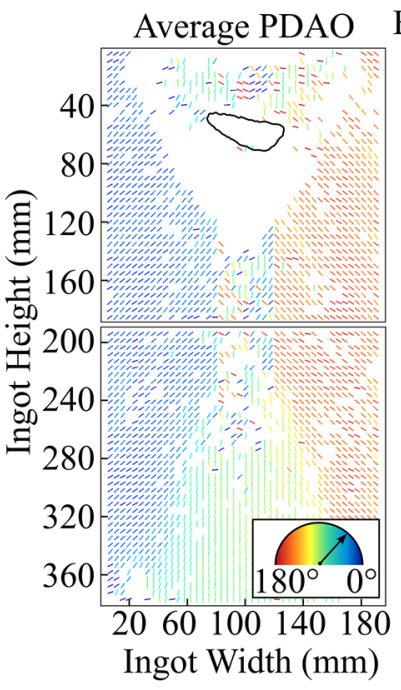

(c)

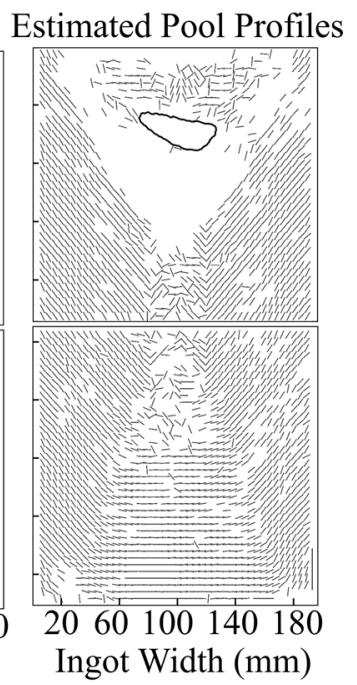

(d)

Fig. 9-(a) Individual primary dendrite arms are identified using particle identification. The color of each primary dendrite arm describes its orientation within the ingot, i.e., its PDAO, according to the legend provided. (b) Melt pool profiles are estimated from the PDAO data in (a) using a streamline function. $(c)$ The average PDAO is calculated from each analysis region using two-point correlation; the PDAO is described by color according to the legend provided. White regions in (c) represent analysis regions where PDAO measurements were not successful. (d) Melt pool profiles are estimated from the PDAO data in (c). These estimates were created by rotating the PDAO lines in (c) by 90 deg. 
toward the center of the ingot where the PDAO is essentially random and the slope of the melt pool profile is expected to change through zero.

Figure 9(c) presents the average PDAO measured from analysis regions using the two-point correlation technique; over 3000 individual analysis regions were studied. Orientations are presented as lines located at the center of each analysis region. The color of each line describes orientation per the legend provided. Empty regions in Figure 9(c) represent analysis regions in which the PDAO could not be determined. Figure 9(c) demonstrates that primary dendrite arm orientations were successfully measured throughout most of the slabs; only the center of the top slab exhibits many regions where PDAO could not be measured. On the left side of the ingot, the PDAO does not vary significantly along most of the ingot height. Variation is slight along the ingot radius until near the center of the ingot. Similar behavior is observed on the right side of the ingot. Along the bottom of the ingot, primary dendrite arms are oriented near $90 \mathrm{deg}$. Regions with apparently random primary dendrite arm orientations are only observed within a narrow strip located in the center of the ingot, where the success rate of PDAO measurement is low because of equiaxed solidification.

Figure 9(d) presents melt pool profile estimates from the two-point correlation technique. The average PDAO measured from each analysis region was simply rotated by $90 \mathrm{deg}$ to estimate the melt pool profile of that region. These estimates break down in the equiaxed center of the ingot where primary dendrite arms are inconsistently oriented. These estimates demonstrate a definite change in shape from the top to the bottom of the ingot. The top of the ingot exhibits a melt pool profile with a "V-shape," which flattens into a "U-shape" toward the bottom of the ingot.

\section{B. Secondary Dendrite Arms}

Figure 10(a) presents the SDAS measurements from particle identification using a Voronoi diagram. A Voronoi diagram represents data in space by forming a polygonal cell around each individual data point. The size and shape of a polygonal cell is chosen such that each position within that cell is located closer to the data point used to create that cell than any other data point. Color represents average SDAS values according to the scale provided. Figure 10(a) demonstrates that SDAS was successfully measured throughout the VAR ingot using particle identification. No trends in these measurements are obvious. Instead, SDAS measurements vary throughout the ingot, and most of these measurements range from 60 to $210 \mu \mathrm{m}$. Figure $10(\mathrm{~b})$ presents the average SDAS values and the corresponding standard deviations for the highlighted regions shown in Figure 10(a), all derived from particle identification. The average SDAS values in the highlighted regions of Figure 10(a) at a given height are the same to within their standard deviations; this is the case for each of the four height positions considered.

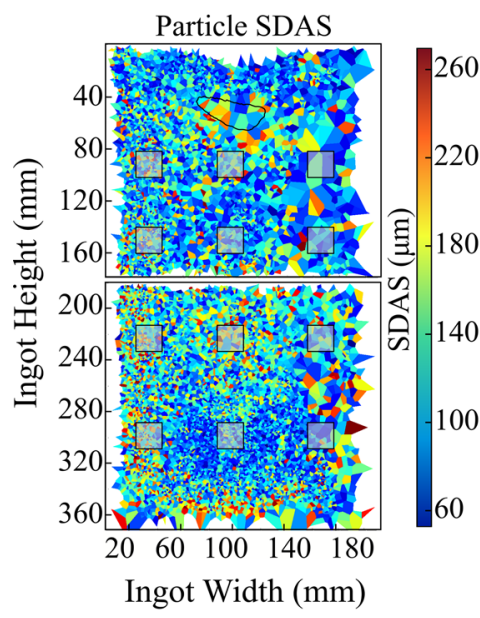

(a)

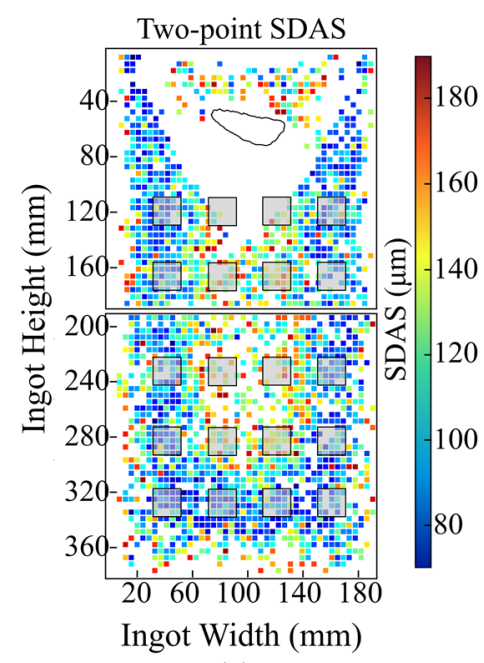

(c)

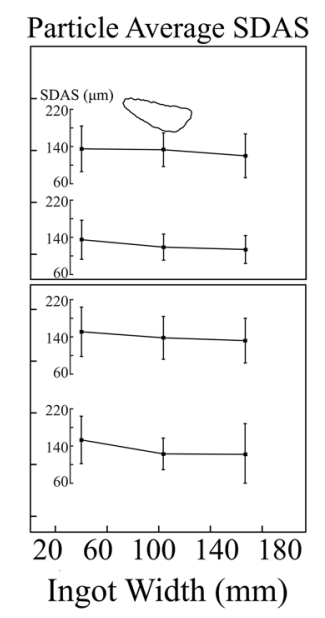

(b)

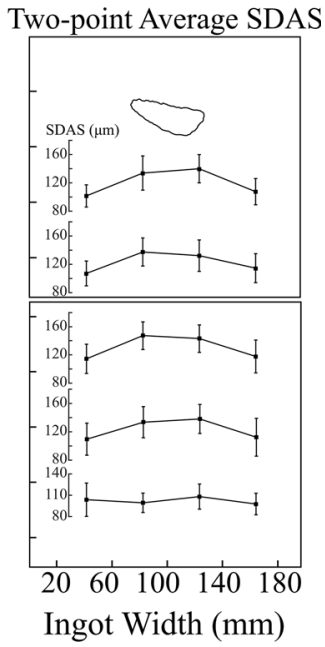

(d)

Fig. 10-(a) SDAS measurements using particle identification are presented as a Voronoi diagram. (b) Average SDAS measurements from the highlighted regions in (a) are presented with the standard deviation of those measurements. (c) The average SDAS determined from each analysis region using two-point correlation is shown. White regions in (c) represent analysis regions where SDAS measurements were not successful. (d) Average SDAS measurements from the highlighted regions in (c) are presented with the standard deviation of those measurements. SDAS values in (a) and (c) are described by their respective color bars. Note that the SDAS data from particle identification and two-point correlation are presented on different scales.

Figure 10(c) presents the SDAS values measured with the two-point correlation technique. Measurements from each analysis region are described by colored squares that represent SDAS values per the scale provided. Empty regions represent analysis regions where SDAS was not measurable. Note that the scale bar describing the SDAS values in Figure 10(c) is narrower than that used in Figure 10(a), indicating the greater precision of the two-point correlation technique. The two-point correlation technique measured SDAS 
values that generally ranged from 80 to $180 \mu \mathrm{m}$. A trend is evident in this SDAS data; measurements are smaller along the sides of the ingot compared to its center. Figure 10(d) presents the average SDAS values and the standard deviations for the highlighted regions shown in Figure 10(c). Again, note that the scale bar used in Figure 10(d) is narrower than that used in Figure 10(b). Figure 10(d) highlights the trend of larger SDAS values at the center of the ingot compared to its sides, based on differences from approximately equal to notably larger than the standard deviations measured. This trend continues until near the bottom of the ingot.

\section{DISCUSSION}

\section{A. Primary Dendrite Arm Orientations and Melt Pool Profiles}

Figure 11 presents (a) the primary dendrite arms determined using particle identification, (b) the associated melt pool profile estimates, (c) the average slope of each melt pool profile, and (d) the electrode current during remelting as a function of ingot height. Because this ingot is axisymmetric about the vertical ingot centerline, only data from the left side of the ingot are presented. The primary dendrite arm orientations measured and presented in Figure 11(a) qualitatively agree with the expected heat flow during solidification. Primary dendrite arms exhibit directional solidification along the ingot sides, where cooling was more rapid than the equiaxed center of the ingot. Few outlier orientations are identified in regions with directional solidification. This suggests that the techniques developed to remove erroneously identified primary dendrite arms were successful. The melt pool profiles qualitatively appear similar throughout the top portion of the ingot and noticeably change only near the bottom of the ingot; see Figure 11(b). This is expected because the bottom of the ingot experiences transient conditions associated with the start of the melt on the water-cooled stool.

The slope of each melt pool profile, Figure 11(c), was calculated by measuring the angle of a straight line drawn between the first and last points of each melt pool profile contained within the highlighted region in Figure 11(b). Analysis was limited to this region because it is affected by neither the surface of the ingot nor the equiaxed center of the ingot. The indicated slope of the melt pool varies significantly along the ingot height. A possible cause of such variation is the effect of transient conditions at the top and bottom of the ingot. Because this ingot is quite small, transient conditions affect much of its remelting. It is unlikely that these changes to the

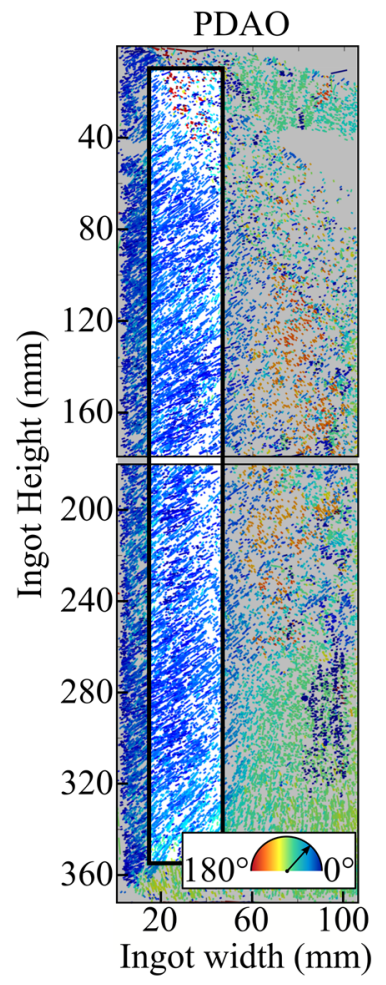

(a)

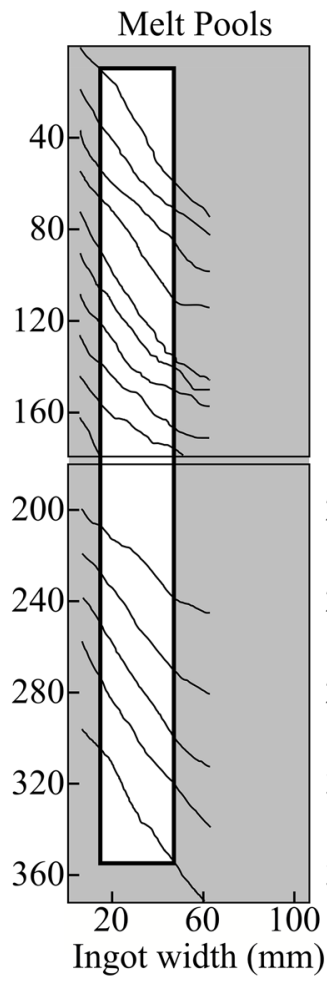

(b)

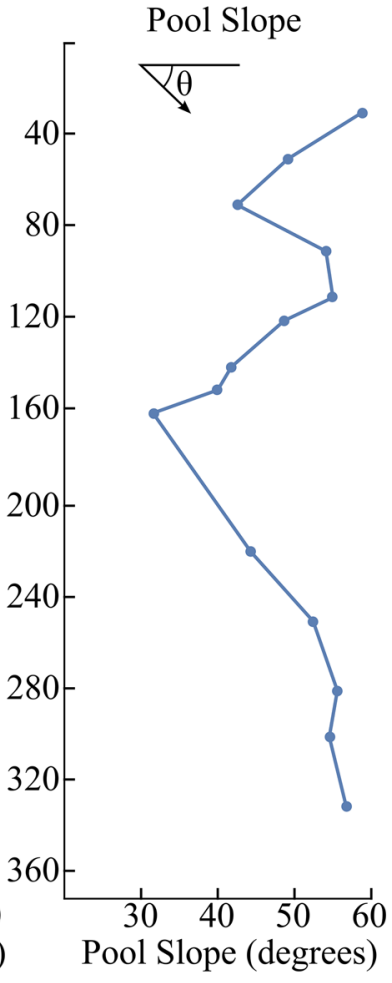

(c)

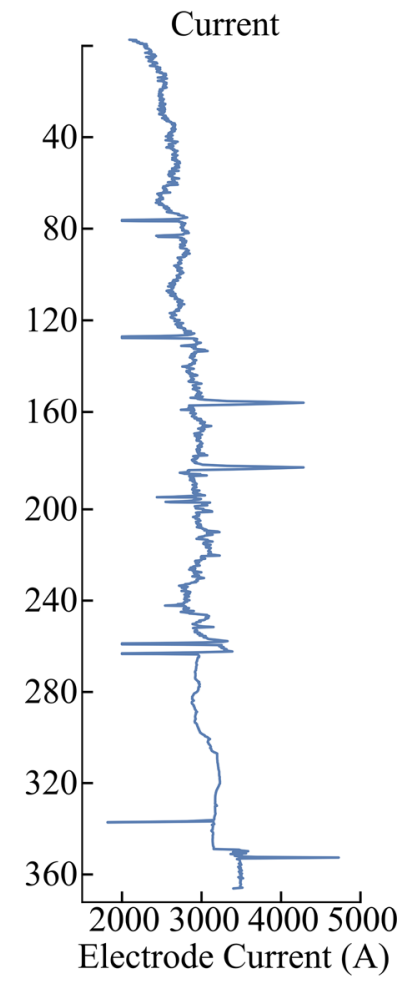

(d)

Fig. 11-(a) Primary dendrite arms identified using particle identification, $(b)$ melt pool profile estimates, and $(c)$ the slope of the melt pool from the left side of the slabs are presented with $(d)$ the electrode current during remelting. Melt pool slopes were calculated from each estimate in the highlighted region in (b). 
slope of the melt pool along the ingot height are related to changes in the electrode current during melting, which is generally constant aside from short spikes; see Figure 11(d). The melt pool profile is only expected to change noticeably with the electrode current for a large change in current held over a significant time. It is suspected that the variations in the slope of the melt pool arise from measurement uncertainty associated with the particle-identification technique and from irregularities along each melt pool profile produced by the streamline estimation methodology.

Figure 12 presents (a) the average PDAO from analysis regions measured using the two-point correlation technique, (b) the associated melt pool profile estimates, (c) the average slope of the melt pool, and (d) the electrode current during remelting as a function of ingot height. Again, only data from the left side of the ingot are presented. Similar to the particle-identification technique, the PDAO is well defined along the sides and bottom of the ingot, where directional solidification occurred, by the two-point correlation technique. The primary dendrite arm orientations in these regions are aligned with the heat flow expected during solidification. The regions of consistent primary dendrite arm orientations penetrate significantly into the ingot, almost reaching its center. Figure 12(a) demonstrates that the two-point correlation technique can measure primary dendrite arm orientations from directionally solidified microstructures, even those with weakly defined orientations near the center of the ingot. This technique, however, was limited in the middle of the top slab where equiaxed solidification occurred because no preferred PDAO exists.

Figure 12(b) shows a change in melt pool shape from a "V-shape" at top to a "U-shape" at bottom. It is not expected that this change was caused by fluctuations in the electrode current but is a natural consequence of the increased thermal conduction in the vertical direction near the bottom of the ingot. Though the shape of the melt pool profile changes along the ingot height, the slope of the melt pool along the sides of the ingot changes little since this is driven by radially dominated thermal conduction into the crucible walls. Figure 12(c) presents the average melt pool slopes from the highlighted regions in Figure 12(b) and their standard deviations. The comparatively small standard deviation in these measurements suggests that this variation in the slope of the melt pool profile with height determined by particle-identification technique, as shown in Figure 11(c), is strongly affected by measurement uncertainty.

Both the particle-identification and the two-point correlation techniques demonstrate an ability to accurately measure the $\mathrm{PDAO}$ in regions of directional solidification. The particle-identification technique is

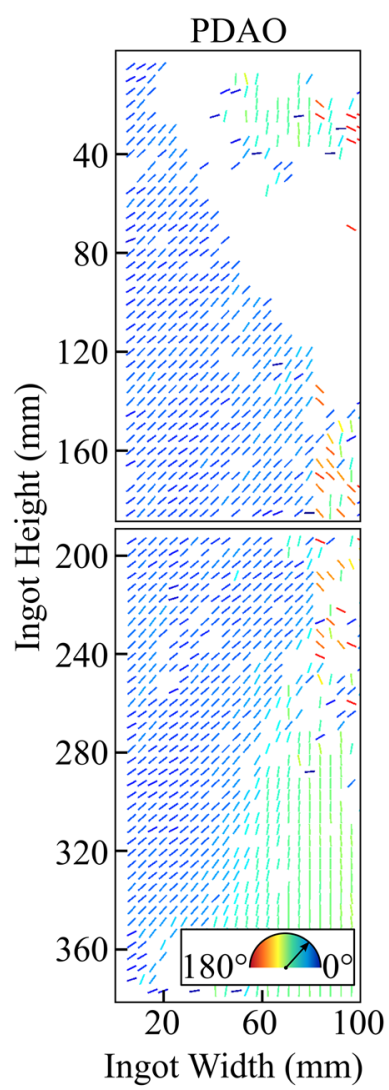

(a)

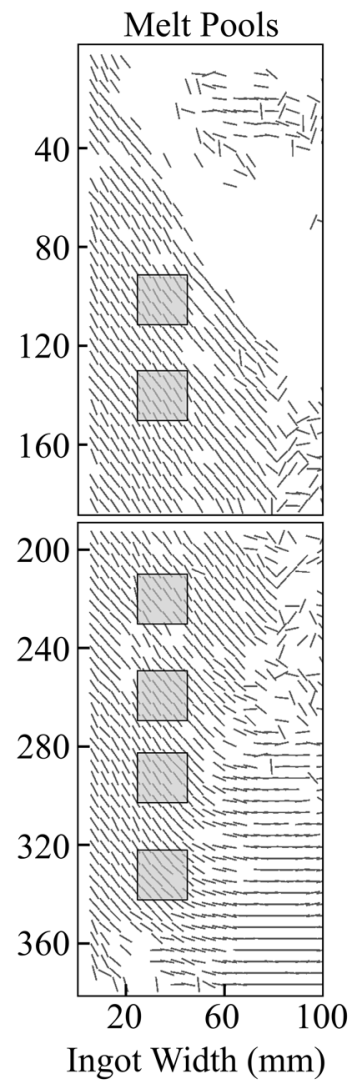

(b)

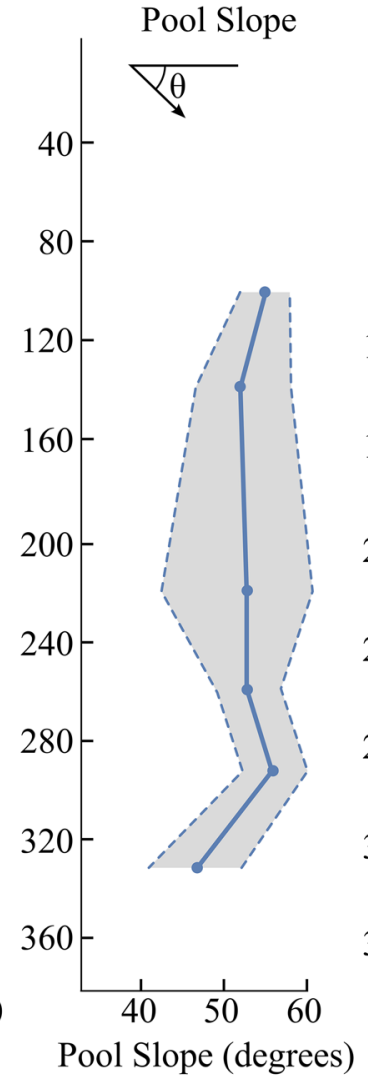

(c)

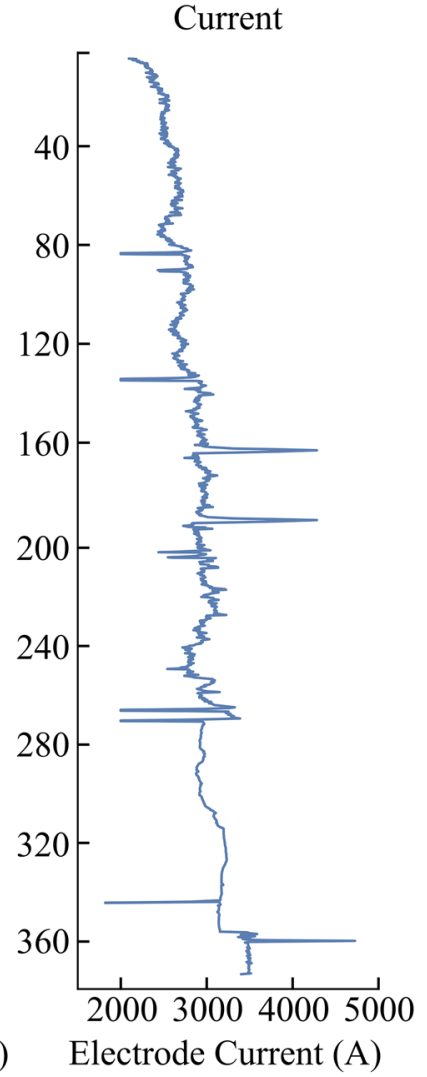

(d)

Fig. 12-(a) Primary dendrite arm orientations calculated using two-point correlation, $(b)$ melt pool profile estimates, and $(c)$ the slope of the melt pool from the left side of the slabs are presented with $(d)$ the electrode current during remelting. The melt pool slopes were calculated from the highlighted regions in (b). 


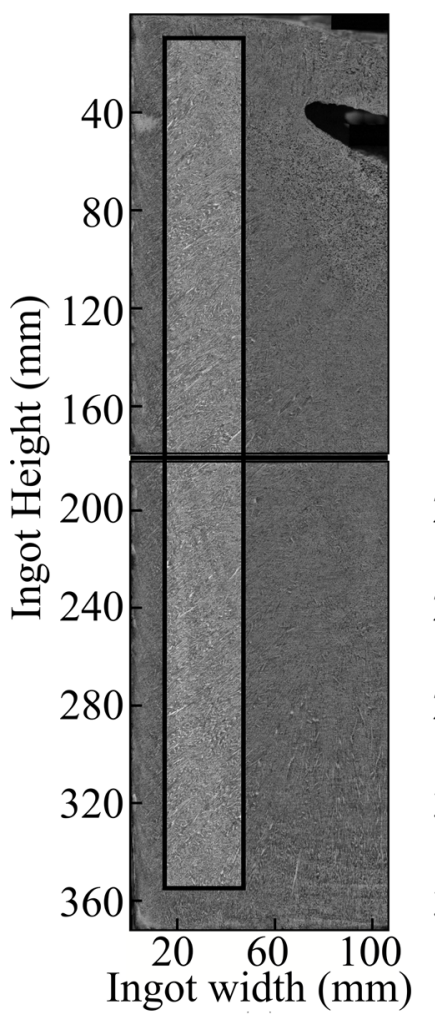

(a)

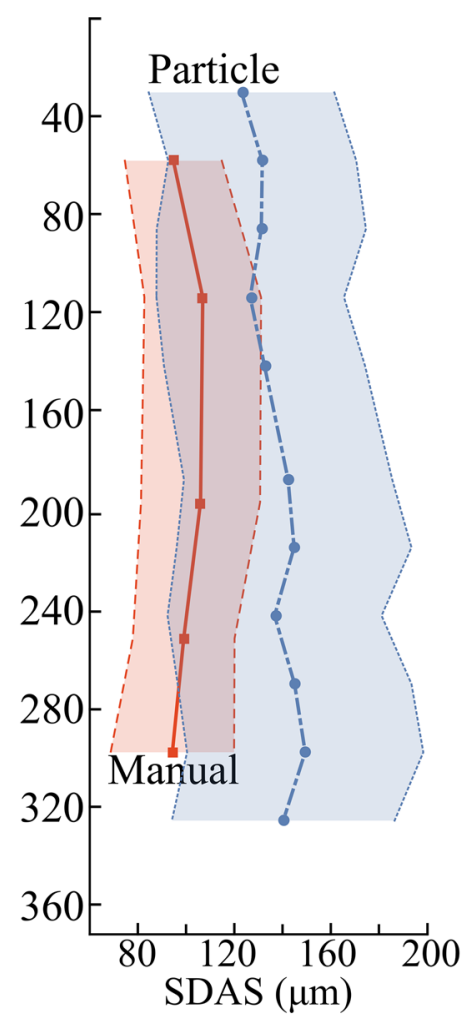

(b)

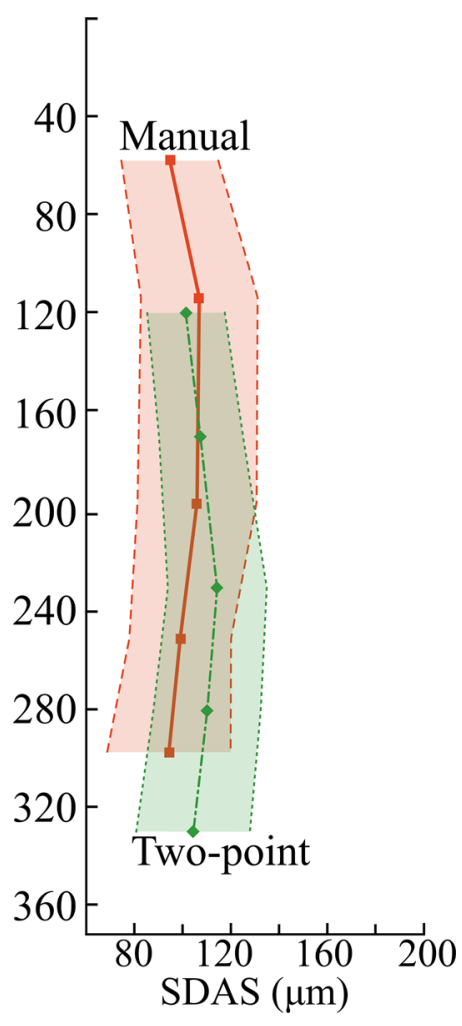

(c)

Fig. 13-(a) Microstructure is presented from the left side of the slabs. Average SDAS measurements and the standard deviations of those measurements were calculated from the highlighted region in (a). Manual SDAS measurements are compared to those from (b) particle identification and $(c)$ two-point correlation.

able to identify some orientations in the equiaxed region where two-point correlation function analysis fails. The data averaging provided by the two-point correlation function produced measurements of average PDAOs that are more easily interpreted and appear more consistent throughout the ingot than results from particle identification. Compare Figures 11 and 12, for example. A change in shape of the melt pool with ingot height, from "V" at the top to "U" at the bottom, is indicated by the two-point correlation technique but is difficult to discern using the particle-identification technique. The average slopes of the melt pool profiles measured along the left side of the VAR ingot are similar for both techniques, approximately $55 \mathrm{deg}$ in Figures 11(c) and 12(c). However, much more local variation in the slope results from the particle-identification technique than the two-point correlation technique. Because melt conditions were consistent during the production of the ingot studied, the melt pool is expected to exhibit a gradual change in shape along the ingot height as observed in the two-point correlation estimates. In contrast, slope estimates from particle identification change from steep at the bottom, to shallow at the middle, and then steep again at the top of the ingot. Subsequent studies are needed to determine if the variations in particle-identification estimates arise from measurement uncertainty or represent an actual phenomenon which is masked by data averaging in the two-point correlation analysis.

Both techniques provide advantages over the pool marker techniques that have been the traditional approach for melt pool measurements to date. Both enable continuous measurement of melt pool profile throughout cross sections of solidified ingots with dendritic microstructures, and neither requires disturbing the melt pool with markers, which may affect local solidification behavior. Uncertainty associated with pool marker interaction with the mushy zone (see Figure 1) is avoided using the techniques developed here. The two-point correlation technique requires less image processing than does particle identification and is thus likely easier to implement in practice. It is of note that the material preparation required for both analysis techniques presented here is similar to that for pool marking methods.

\section{B. Secondary Dendrite Arm Spacings}

SDAS measurements from particle identification vary significantly across the slabs, and no trends in these measurements are obvious, as demonstrated by Figure 10(a). Two plausible explanations for the lack 


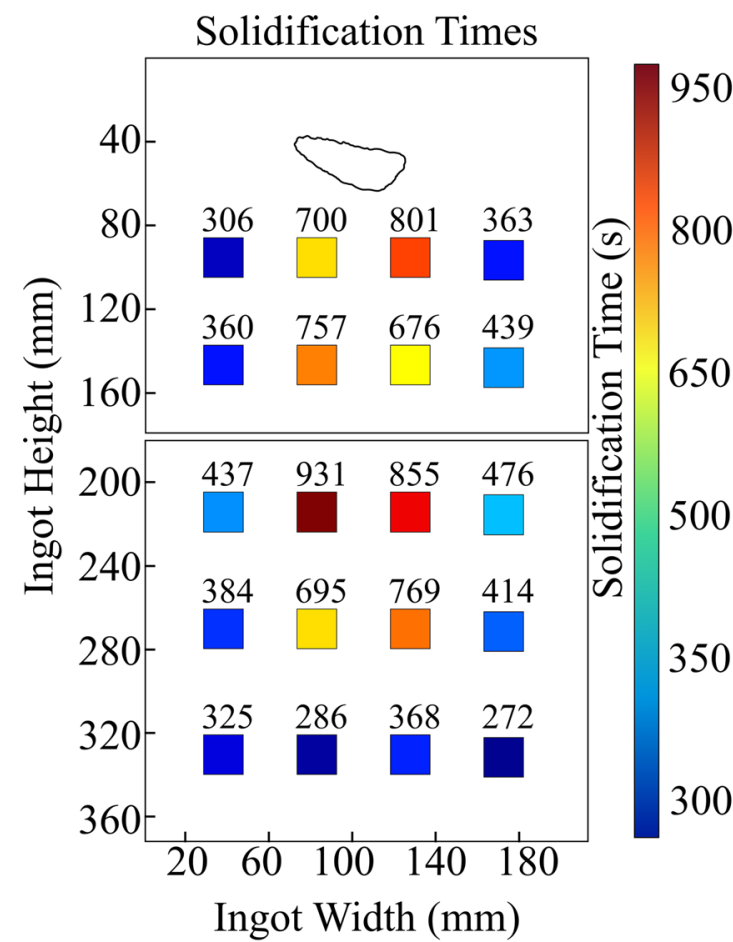

(a)

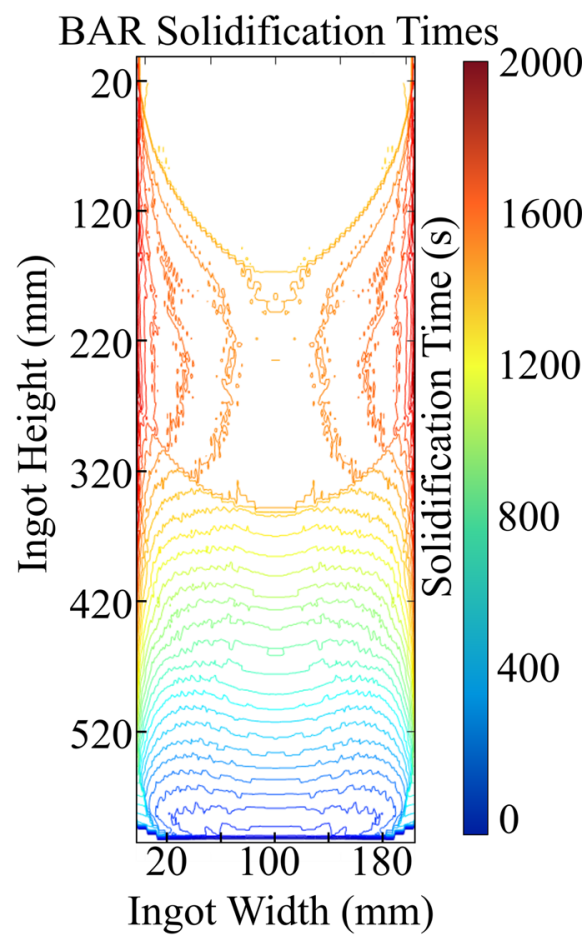

(b)

Fig. 14-(a) Solidification times calculated using Eq. [1] from secondary dendrite arm spacing (SDAS) measurements by two-point correlation are provided from the highlighted regions in Fig. 10(c). (b) Solidification times predicted using the Basic Axisymmetric Remelting (BAR) code ${ }^{[46]}$ are presented. The ingot used for the BAR predictions is taller than the ingot characterized in this study.

of trends in SDAS values were identified. First, SDAS may indeed vary indiscriminately throughout the ingot. This explanation is unlikely because solidification times, and thus SDAS values, are expected to differ between regions of directional and equiaxed solidification. Second, the automated measurement technique may lack the sensitivity necessary to reveal differences in SDAS with position. For example, if an identified particle is not precisely aligned with its primary dendrite arm, the SDAS measurement will be adversely affected. This scenario leads to a larger SDAS measurement because some secondary dendrite arms are missed, i.e., not counted, during analysis. This can lead to measurement uncertainties larger than the changes in SDAS between locations within the ingot, thus masking real differences; see Figure 10(b).

In contrast to the particle-identification technique, a trend in the SDAS data is evident using two-point correlation. SDAS values are smaller along the sides of the ingot and larger in the center; see Figures 10(c) and (d). These data are also axisymmetric about the vertical ingot centerline. Because the VAR ingot cools more slowly at its center compared to its sides, SDAS values are expected to be larger in the center. That said, because this SDAS measurement technique relies on an accurate measure of the PDAO, results may be misleading if the PDAO is not accurately established. The uncertainty potentially introduced by this effect could not be quantified in this investigation.
SDAS measurements were more precise from twopoint correlation than particle identification. SDAS measurements typically ranged from 80 to $180 \mu \mathrm{m}$ for two-point correlation and from 60 to $210 \mu \mathrm{m}$ for particle identification. The accuracies of SDAS measurements produced by particle identification and two-point correlation were determined by comparisons to manual measurements. Figure 13(a) highlights the region on the left side of the ingot where average SDAS values were calculated using both techniques. SDAS values were manually measured in this same region and are compared to those from particle identification in Figure 13(b) and two-point correlation in Figure 13(c). SDAS measurements from particle identification are consistently larger than manual measurements by a factor of approximately 1.3 , as demonstrated in Figure 13(b). This suggests that the particle-identification technique is missing some secondary dendrite arms, which leads to an error that increases the spacing measurement. The standard deviation of SDAS measurements from particle identification is much larger than that from manual measurements. This confirms that the large measurement uncertainty with the particle-identification technique is masking any real changes in SDAS values. The SDAS measurements from the two-point correlation technique are quite similar to the manual measurements, as demonstrated in Figure 13(c). The standard deviations are also similar. These data indicate that the two-point correlation technique 
provides SDAS measurements that are effectively as accurate as manual measurements, the current standard for best practice.

Both particle-identification and two-point correlation SDAS measurement techniques provide advantages over manual measurements. These new techniques allow for measurement of SDAS continuously throughout ingot cross-sectional slabs, and these measurements can be used to improve and validate computational models. Additionally, these automated measurements can be acquired faster than manual measurements. Note that the same amount of material preparation is required for the automated techniques developed here as for manual measurement of SDAS.

Manual SDAS measurements reported in the literature from alloy 718 ingots produced by $\mathrm{VAR}^{[69]}$ and $\mathrm{ESR}^{[44]}$ are compared to those acquired from two-point correlation in this study. These comparisons are used to illustrate the effect of ingot size on SDAS and to provide perspective for the SDAS values presented here. Melt conditions for ingots in the extant literature, which are not reported in detail, may differ from the ingot in this study and could impact the SDAS comparisons presented here. Patel and Murty report SDAS measurements for an ingot produced by VAR with a diameter similar to the one investigated here. SDAS ranges from $92 \mu \mathrm{m}$ near ingot sides to $120 \mu \mathrm{m}$ near the ingot center. ${ }^{[69]}$ These measurements generally agree with those reported here. SDAS measurements reported in the literature for industrial-scale (diameters $>50 \mathrm{~cm}$ ) alloy 718 ingots produced by VAR range from $80 \mu \mathrm{m}$ along the ingot sides to $225 \mu \mathrm{m}$ near the ingot center. ${ }^{[69]}$ Similarly, SDAS measurements for industrial-scale alloy 718 ingots produced by ESR range from $110 \mu \mathrm{m}$ along the ingot sides to $160 \mu \mathrm{m}$ near the ingot center. ${ }^{[4]}$ SDAS values are similar for large and small ingots along the sides of the ingot because heat transfer rates near the crucible wall are comparable. In the center of large ingots, cooling is typically much slower than in small ingots.

Local solidification times were calculated with Eq. [1] using the SDAS measurements produced from the two-point correlation technique. The average solidification times calculated from the highlighted regions in Figure 10(c) are provided in Figure 14(a). Solidification time at the side of the ingot, approximately 40 and 160 $\mathrm{mm}$ from the left surface of the ingot, is $377 \pm 65 \mathrm{~s}$. The solidification time at the center of the ingot, taken as approximately 80 and $120 \mathrm{~mm}$ from the left surface of the ingot, is $684 \pm 204 \mathrm{~s}$. The solidification time near the water-cooled stool at the bottom of the ingot, approximately $330 \mathrm{~mm}$ from the top of the ingot, is 312 $\pm 43 \mathrm{~s}$. Using the SDAS values reported in the literature, local solidification times for a similarly sized alloy 718 ingot produced by VAR can be calculated. These times, calculated as $227 \mathrm{~s}$ along the side and $505 \mathrm{~s}$ in the center of the ingot, ${ }^{[69]}$ compare reasonably well with local solidification times presented in this study. For large industrial-sized ingots produced by VAR and ESR, solidification times calculated from SDAS values near the sides of these ingots are 150 and $389 \mathrm{~s}$, respectively. ${ }^{[4,69]}$ These solidification times are similar to the small ingot because they are close to the crucible walls where heat transfer from the ingot is similar. In the center of the ingot, solidification times calculated from SDAS measurements are $3328 \mathrm{~s}$ for the industrial-sized VAR ingot ${ }^{[69]}$ and $1197 \mathrm{~s}$ for the ESR ingot. ${ }^{[44]}$ As expected, these values are much larger than the small ingot because the centers of the large ingots cannot cool as quickly.

Solidification times calculated from SDAS measurements are compared in Figure 14 to those predicted using the Basic Axisymmetric Remelting (BAR) code. ${ }^{[46]}$ The BAR code uses finite volume methods to model solidification during remelting processes such as VAR assuming an axisymmetric ingot geometry and steadystate remelting conditions. Solidification times from the BAR code are presented in Figure 14(b) for an alloy 718 ingot produced by VAR using processing conditions similar to the ingot characterized in this study. Simulation results for exactly the ingot characterized here were not available. The ingot used for the BAR predictions is identical in diameter but approximately twice the height of the ingot investigated in this study and was produced under similar processing conditions. Results from the bottom half of the simulation, from approximately 300 to $620 \mathrm{~mm}$ along the ingot height in Figure 14(b), can be compared to the solidification times estimated from SDAS measurements in Figure 14(a). Note that the automated SDAS measurement techniques developed here make this comparison practical. These techniques, or others like them, can be used to improve development and assessment of computational models for remelting.

For an equivalent location in each ingot, solidification times predicted using the BAR code typically range between 100 and 900 s longer than those calculated from SDAS measurements. The model accurately predicts short solidification times along the bottom of the ingot but consistently predicts longer solidification times along the sides of the ingot. Additionally, the U-shape observed in the solidification times calculated from SDAS measurements is not predicted by the BAR code. Several factors may contribute to the discrepancies between simulation and measurement. First, discrepancies likely arise because the BAR code was run on a taller ingot geometry than the one characterized. Second, ingot shrinkage predicted by the BAR model may overestimate the reduction in heat transfer between the ingot and chilled crucible walls. Third, the BAR code assumes steady-state remelting conditions, and both the ingot modeled and the one characterized likely never reached steady-state conditions because of their short heights.

\section{CONCLUSIONS}

Solidification microstructures in two slabs extracted from the midline cross-section of a nickel alloy 718 ingot produced by vacuum arc remelting (VAR) were imaged using automated macrophotography. A digital image montage was created for the prepared surface of each slab, covering its entire area. Dendritic features were measured throughout these montage images using 
automated particle-identification and two-point correlation techniques. The following conclusions are drawn from the reported results.

1. Automated macrophotography provides a practical means of imaging large areas of etched ingot slabs with resolution sufficient to discern primary and secondary dendrite arms. Individual macrophotographs can be digitally stitched into montage images covering entire ingot slab surfaces. This capability was demonstrated for two alloy 718 cross-sectional slab surfaces of approximately $400 \mathrm{~cm}^{2}$ in area.

2. Primary dendrite arms and their orientations were identified from digital image montages using a particle-identification technique. This technique identified 52 pct of the primary dendrites that were manually identified. It was demonstrated that primary dendrite arm orientation (PDAO) measurements made by this technique can be used to reconstruct melt pool profiles that agree with those expected during the VAR process.

3. Primary dendrite arms were also identified and their orientations measured from digital image montages using a two-point correlation technique. It was demonstrated that PDAO measurements made by this technique can be used to reconstruct melt pool profiles more reliably in regions of directional solidification than by the particle-identification technique.

4. Both the particle-identification and two-point correlation techniques successfully measured secondary dendrite arm spacing (SDAS) throughout the ingot cross-sectional slabs, except in regions of primarily equiaxed solidification. The two-point correlation technique proved to be the more accurate and precise of the two techniques when checked against manual measurements of SDAS.

5. Solidification times were calculated from SDAS measurements made using the two-point correlation technique. Solidification time near the outer diameter was calculated as $377 \pm 65 \mathrm{~s}$, and solidification time near the ingot center was calculated as $684 \pm 204 \mathrm{~s}$.

6. Implementation of the particle-identification technique required some processing of the initial microstructural images and significant filtering of data, all of which required tuning of many parameters particular to the specific image data set. The two-point correlation technique required only grayscale image normalization and thresholding prior to analysis and required tuning of only a few parameters to the particular image data set, principally analysis region size and transportable window size. Because of this, it is expected that the two-point correlation technique will be more robust and easier to implement in practice than the particle-identification technique.

7. The particle-identification and two-point correlation techniques provide significant advantages over the pool marker techniques that to date have been the standard for melt pool measurements. Both enable continuous measurement of PDAO throughout cross sections of solidified ingots with dendritic microstructures, particularly for strongly directional solidification, and neither requires disturbing the melt pool with markers to measure PDAO. Both techniques provide time savings over the manual measurement of SDAS. Both techniques provide continuous measurement of SDAS throughout cross sections of solidified ingots.

\section{ACKNOWLEDGMENTS}

The authors would like to acknowledge support from individuals at Allegheny Technologies Inc., Carpenter Technology Corp., and Special Metals Corp., especially David G. Evans (Special Metals Corp.) for his assistance with material preparation. The material investigated in this study was produced under funding from the Office of Naval Research (ONR) through contract numbers N00014-11-1-0366 and N0001412-1-0811. Funding for this work was provided by the Air Force Research Laboratory (AFRL) through the Metals Affordability Initiative (MAI) under contract number FA8650-11-2-5224-B.

\section{OPEN ACCESS}

This article is distributed under the terms of the Creative Commons Attribution 4.0 International License (http://creativecommons.org/licenses/by/4.0/), which permits unrestricted use, distribution, and reproduction in any medium, provided you give appropriate credit to the original author(s) and the source, provide a link to the Creative Commons license, and indicate if changes were made.

\section{REFERENCES}

1. J.F. Barker: ed. E.A. Loria, Superalloy 718: Metallurgy and Applications, 1989, pp. 269-77.

2. D.F. Paulonis and J.J. Schirra: ed. E.A. Loria, Superalloys 718 , 625, 706 and Various Derivatives, 2001, pp. 13-23.

3. R.E. Schafrik, D.D. Ward and J.R. Groth: ed. E.A. Loria, Superalloys 718, 625, 706 and Various Derivatives, 2001, pp. 1-11.

4. R.C. Reed: The Superalloys Fundamentals and Applications, Cambridge University Press, Cambridge, 2006.

5. M.J. Donachie and S.J. Donachie: Superalloys: A Technical Guide, 2nd ed., ASM International, Geauga, 2002.

6. T.M. Pollock and S. Tin: J. Propul. Power, 2006, vol. 22, pp. 36174.

7. J.F. Radavich: ed. E.A. Loria, Superalloy 718: Metallurgy and Applications, 1989, pp. 229-40

8. M.C. Chaturvedi and Y. Han: ed. E.A. Loria, Superalloy 718: Metallurgy and Applications, 1989, pp. 489-98.

9. A.H. Jones: ed. E.A. Loria, Superalloy 718: Metallurgy and Applications, 1989, pp. 307-18.

10. Y. Wang, W.Z. Shao, L. Zhen, L. Yang and X. M. Zhang: Mater. Sci. Eng. A, 2008, vol. 497, pp. 479-86, 2008.

11. C. Malara and J. Radavich: ed. E.A. Loria, Superalloys 718, 625, 706 and Various Derivatives, 2005, pp. 25-33.

12. A. Lingenfelter: ed. E.A. Loria, Superalloy 718: Metallurgy and Applications, 1989, pp. 673-83.

13. S.D. Antolovich: ed. E.A. Loria, Superalloy 718: Metallurgy and Applications, 1989, pp. 647-53. 
14. M.J. Cieslak, G.A. Knorovsky, T.J. Headley and A.D. Romig Jr.: ed. E.A. Loria, Superalloy 718: Metallurgy and Applications, 1989, pp. 59-67.

15. J.A. Schey: Introduction to Manufacturing Processes, McGraw-Hill Inc., New York, 1997.

16. Heat Treater's Guide: Practices and Procedures for Nonferrous Alloys, 2nd ed., H. Chandler, ed., Heat Treater's Guide: Practices and Procedures for Nonferrous Alloys, ASM International, Geauga, 1996.

17. N.A. Wilkinson: ed. E.A. Loria, Superalloy 718: Metallurgy and Applications, 1989, pp. 119-33.

18. M.C. Mataya and D.K. Matlock: ed. E.A. Loria, Superalloy 718 : Metallurgy and Applications, 1989, pp. 155-78.

19. P.E. Mosser, G. Leconte, J. Leray, A. Lasalmonie and Y. Honnorat: ed. E.A. Loria, Superalloy 718: Metallurgy and Applications, 1989, pp. 179-88.

20. R.G. Carlson and J.F. Radavich: ed. E.A. Loria, Superalloy 718 : Metallurgy and Applications, 1989, pp. 79-95.

21. R.M. Forbes Jones and L.A. Jackman: JOM, 1999, vol. 51, pp. 27-31.

22. S.M. Jones, J. Radavich and S. Tian: ed. E.A. Loria, Superalloy 718: Metallurgy and Applications, 1989, pp. 589-97.

23. J.L. Burger, R.R. Biederman and W.H. Couts: ed. E.A. Loria, Superalloy 718: Metallurgy and Applications, 1989, pp. 207-17.

24. J.M. Poole, K.R. Stultz and J.M. Manning: ed. E.A. Loria, Superalloy 718: Metallurgy and Applications, 1989, pp. 219-28.

25. J.M. Moyer, L.A. Jackman, C.B. Adasczik, R.M. Davis and R. Forbes Jones: ed. E. A. Loria, Superalloys 718, 625, 706 and Various Derivatives, 1994, pp. 39-48.

26. L.A. Jackman: JOM, 1998, vol. 50, pp. 26-29.

27. A. Mitchell: ed. E.A. Loria, Superalloy 718: Metallurgy and Applications, 1989, pp. 1-15.

28. F.J. Zanner, R.L. Williamson, R.P. Harrison, H.D. Flanders, R.D. Thompson and W.C. Szeto: ed. E.A. Loria, Superalloy 718 . Metallurgy and Applications, 1989, pp. 17-32.

29. K.O. Yu and J.A. Domingue; ed. E.A. Loria, Superalloy 718 : Metallurgy and Applications, 1989, pp. 33-48.

30. W. Kurz and D.J. Fisher: Fundamentals of Solidification, 4th ed., Trans Tech Publications Inc, 1998.

31. A. Wagner, B.A. Shollock, and M. McLean: Mater. Sci. Eng. A, 2004, vol. 374, pp. 270-79.

32. Y.Z. Zhou, A. Volek, and N.R. Green: Acta Mater., 2008, vol. 56, pp. 2631-37.

33. J.A. Van Den Avyle, J.A. Brooks, and A.C. Powell: JOM, 1998, vol. 50 , pp. $22-25$.

34. W.J. Boettinger, S.R. Coriell, A.L. Greer, A. Karma, W. Kurz, M. Rappaz, and R. Trivedi: Acta Mater., 2000, vol. 48, pp. 43-70.

35. L.A. Jackman, G.E. Maurer and S. Widge: ed. E.A. Loria, Superalloys 718, 625, 706 and Various Derivatives, 1994, pp. 153-66.

36. A. Soller, A. Jardy, R. Larue, D. Ablitzer and G. Reiter: ed. P. D. Lee, in Proceedings of the 2005 International Symposium on Liquid Metal Processing and Casting, 2005, pp. 39-48.

37. F. Shved: eds. P.D. Lee, A. Mitchell and R. Williamson, in Proceedings of the 2009 International Symposium on Liquid Metal Processing and Casting, 2009, pp. 85-93.

38. P. Auburtin, T. Wang, S.L. Cockcroft, and A. Mitchell: Metall. Mater. Trans. B, 2000, vol. 31B, pp. 801-11.

39. T. Antonsson and H. Fredriksson: Metall. Mater. Trans. B, 2005, vol. 36B, pp. 85-96.

40. X. Wang, M. Wark, M. Jacobs, and M. Barratt: Metall. Mater. Trans. A, 2008, vol. 39A, pp. 2981-89.

41. F. Zanner, R. Williamson and R. Erdmann: ed. P.D. Lee, in Proceedings of the 2005 International Symposium on Liquid Metal Processing and Casting, 2005, pp. 13-27.

42. K. Morita, T. Suzuki, T. Taketsuru, D.G. Evans and W. Yang: ed. E.A. Loria, Superalloys 718, 625, 706 and Various Derivatives, 2001, pp. 149-60.
43. A. Kermanpur, D.G. Evans, R.J. Siddall, P.D. Lee, and M. McLean: J. Mater. Sci., 2004, vol. 39, pp. 7175-82.

44. C. O'Connell, J. deBarbadillo, D. Evans, R. Minisandram, R. Smith, J. Yanke, E. Taleff, T. Ivanoff, K. Kelkar, W. Hutchison and M. Benedict: in Liquid Metal Processing \& Casting Conference 2017, 2017.

45. R.L. Williamson, J.J. Beaman, F.J. Zanner and J.J. DeBarbadillo: ed. E.A. Loria, Superalloys 718, 625, 706 and Various Derivatives, 2005, pp. 47-56.

46. L.A. Bertram, P.R. Schunk, S.N. Kempka, F. Spadafora, and R. Minisandram: JOM, 1998, vol. 50, pp. 18-21.

47. L. Nastac: Metall. Mater. Trans. B, 2014, vol. 45B, pp. 44-50.

48. L. Nastac: Mater. Sci. Technol., 2012, vol. 28, pp. 1006-13.

49. L. Nastac: Acta Mater., 1999, vol. 47, pp. 4253-62.

50. L. Nastac, S. Sundarraj, K.-O. Yu, and Y. Pang: JOM, 1998, vol. 50, pp. 30-35.

51. R.L. Williamson, M.E. Schlienger, C.L. Hysinger and J.J. Beaman: ed. E.A. Loria, Superalloys 718, 625, 706 and Various Derivatives, 1997, pp. 37-46.

52. X. Xu, R.M. Ward, M.H. Jacobs, P.D. Lee, and M. McLean: Metall. Mater. Trans. A, 2002, vol. 33A, pp. 1795-804.

53. X. Xu, W. Zhang, and P.D. Lee: Metall. Mater. Trans. A, 2002, vol. 33A, pp. 1805-15.

54. R.C. Atwood and P.D. Lee: J. Mater. Sci., 2004, vol. 39, pp. 719397.

55. E.N. Kondrashov, M.I. Musatov, A.Y. Maksimov, A.E. Goncharov, and L.V. Konovalov: J. Eng. Thermophys., 2007, vol. 16, pp. 19-25.

56. M. Rappaz and W.J. Boettinger: Acta. Mater., 1999, vol. 47, pp. 3205-19.

57. W.J. Boettinger, U.R. Kattner, S.R. Coriell, Y.A. Chang and B.A. Mueller: eds. M. Cross and J. Campbell, Modeling of Casting, Welding and Advanced Solidification Processes, VII, 1995, pp. $649-56$.

58. T.J. Watt, E.M. Taleff, F. Lopez, J. Beaman and R. Williamson: in Proceedings of the 2013 International Symposium on Liquid Metal Processing \& Casting, 2013, pp. 261-70.

59. T. Le Roux and D.A. Wells: ed. E.A. Loria, Superalloy 718: Metallurgy and Applications, 1989, pp. 109-18.

60. T.A. Ivanoff: Reconstruction of Solidification History from Cast Microstructure in Remelted Nickel Alloy 718, Doctoral Dissertation, The University of Texas at Austin, 2017.

61. J. Schindelin, I. Arganda-Carreras, E. Frise et al:: Nat. Methods, 2012, vol. 9, pp. 676-82.

62. S.M. Pizer, E.P. Amburn, J.D. Austin, R. Cromartie, A. Geselowitz, T. Greer, B.T.H. Romeny, J.B.Zimmerman and K. Zuiderveld: Computer Vision, Graphics, and Image Processing, 1987, vol. 39, pp. 355-68.

63. MATLAB R2015a version 8.5.0, Natick, MA: MathWorks Inc., 2015.

64. C. Jung-Kuei, The Effect of Microstructure on Cavitation during Hot Deformation in Fine-grained AA5083 Aluminum Alloy Sheet Material, Doctoral Dissertation, The University of Texas at Autsin, 2008.

65. A. Tewari, A.M. Gokhale, J.E. Spowart, and D.B. Miracle: Acta Mater., 2004, vol. 52, pp. 307-19.

66. A.M. Gokhale, A. Tewari, and H. Garmestani: Scr. Mater., 2005, vol. 53, pp. 989-93.

67. Mathematica 10.1, Champaign, IL: Wolfram Research, Inc., 2015.

68. R.B. Blackman and J.W. Tukey: Bell Syst. Tech. J., 1958, vol. 37, pp. 185-282.

69. A.D. Patel and Y.V. Murty: ed. E.A. Loria, Superalloys 718, 625, 706 and Various Derivatives, 2001, pp. 123-32.

Publisher's Note Springer Nature remains neutral with regard to jurisdictional claims in published maps and institutional affiliations. 